\title{
An Efficient Underwater Acoustical Localization Method Based On Time Difference and Bearing Measurements
}

\author{
Liang Zhang, Tao Zhang, Hyo-Sang Shin, and Xiang Xu
}

\begin{abstract}
This paper addresses the underwater acoustical localization problem by using the time-difference-of-arrival (TDOA) and bearing-angle-of-arrival (BAOA) measurements. For the underwater acoustic equipment, such as the ultra-short baseline system (USBL), whose bearing measurements are different from the traditional angle-of-arrival (AOA) model, a closed-form solution for the hybrid TDOA/BAOA-based source localization problem is developed. However, the solution suffers from the measurement noise and cannot achieve the Cramer-Rao lower bound (CRLB) performance in the case of large measurement noise. Thus, an iterative constrained weighted least squares method is presented to further minimize the error in the case of large noise. The CRLB for hybrid TDOA/BAOA source localization is analyzed and the solution is proved to achieve the CRLB performance. Numerical simulations and field tests demonstrate that the proposed method outperforms the traditional methods in terms of estimation bias and accuracy. It can achieve the CRLB performance better.
\end{abstract}

Index Terms-USBL, TDOA, BAOA, Bearing, iterative constrained weighted least squares, CRLB

\section{INTRODUCTION}

Source localization is a classical subject due to its importance in the applications of sensor networks, radar, and underwater navigation ${ }^{[1][2]}$. In such applications, the main idea of source localization is to use the noisy measurement, such as the timedifference-of-arrival (TDOA $)^{[3][4][5][6]}$, time-of-arrival (TOA) $)^{[7]}$ ${ }^{[8]}$, and angle-of-arrival (AOA) ${ }^{[9][10]}$.

TDOA-based localization problem has the advantages of no needing the synchronize sensor clocks with that of the target. Especially in the aspects of single-source passive navigation ${ }^{[2]}$ or the underwater acoustical localization of the black box ${ }^{[11]}$, the clock synchronization error is not known and the TDOAbased localization method is often more effective than the TOA-based localization method. Chan proposed a two-stage weighted least squares (TWLS) algorithm for the TDOA-based

Manuscript received xxxx, xxxx; revised xxxx, xxxx; accepted xxxx, xxxx. This work was supported in part by National Natural Science Foundation of China 52071080, the Inertial Technology Key Lab Fund under Grant 614250607011709 ,in part by the Fundamental Research Funds for the Central Universities under Grant 2242020k1G009, Key Laboratory Fund for Underwater Information and Control under Grant 614221805051809, and in part by the Jiangsu Key Laboratory Fund for Green Ship Technology under Grant 2019Z01, Remaining funds cultivation project of National Natural Science Foundation of Southeast University under Grant 9S20172204. localization problem ${ }^{[12]}$. It is well known for its low computational complexity and proven approximate efficiency. Several improved algorithms have been proposed ${ }^{[13][14]}$. AOAbased localization problem does not need accurate clock synchronization between sensors and the closed-form solution was developed ${ }^{[15][16]}$. Recently, the hybrid systems that combine two or more noisy measurements have drawn considerable attention. Li developed a two-step least-square location estimator for the TDOA/AOA location scheme for wideband code-division multiple-access wireless communication systems ${ }^{[17]}$. A geometrically constrained optimization approach was applied to the hybrid TDOA/AOA location scheme to improve the localization accuracy ${ }^{[18]}$. In 2016, a simple TDOA/AOA method using two stations was developed and the method could be extended to more than two stations ${ }^{[19]}$. When the prior knowledge about the source range is not available, an iterative maximum likelihood estimator (MLE) for the source location under modified polar representation (MPR) was developed ${ }^{[20]}$. Note that all of the above-mentioned methods were presented for the source localization problem, in which the azimuth and elevation angles are considered in the AOA model. The closed-form solutions in these methods are designed for the traditional TDOA/AOA model. In particular, azimuth is the angle between the projection of slant distance, which is the distance between the source and the sensor, onto a horizontal plane and the $\mathrm{X}$-axis ${ }^{[20]}$. The elevation angle is the angle between the projection of slant distance onto a horizontal plane and the slant distance ${ }^{[20]}$. This AOA model requires both of the azimuth and elevation angles to complete the positioning. However, when the underwater sensor consists of multiple hydrophones, such as the USBL, the AOA measurements are available ${ }^{[2]}$ and they can be applied to the localization problem. The new underwater AOA model is based on the bearing measurements. In order to distinguish from the traditional AOA model, it is named as the bearing angle of arrival (BAOA). The

(Corresponding author: Tao Zhang.) L. Zhang, T. Zhang are with the School of Instrument Science and Engineering, the Key Laboratory of Micro-Inertial Instrument and Advanced Navigation Technology, Ministry of Education, Southeast University, Nanjing 21 0096, China (e-mail: zhangliang418@seu.edu.cn; zhangtao22@seu.edu.cn;); H.-S. Shin is with the Institute of Aerospace Sciences, SATM, Cranfield University, MK43 0AL Cranfield,U.K., E-mail: (h.shin@ cranfield.ac.uk); Xiang Xu is with the School of Electronic and Information Engineering, Soochow University, Suzhou 215006, China (e-mail: hsianghsu@ 163.com) 
bearing angles studied in the paper are the angles between the slant distance and the $\mathrm{x}$-axis (bearing $\alpha$ ) and the $\mathrm{y}$-axis (bearing $\beta)^{[21]}{ }^{[22]}$. Bearing $\alpha$ or bearing $\beta$ alone can complete the positioning. Since the USBL can output two bearing angles, both of the two bearing angle are used in the localization problem in the paper. Different definitions of the AOA model will lead to different algebraic expressions of the closed solutions. Thus, the above algebraic expressions of the closed solutions are not suitable for the new model in the manuscript. To the best of our knowledge, there is not yet a sufficiently effective solution for the new underwater acoustical localization model. Thus, one of the tasks of this paper is to derive a new algebraic expression of a closed solution based on the new BAOA model. Besides, the location bias caused by the noisy measurement will be considered in the paper. However, it is not considered in the above traditional TDOA/AOA localization model.

For the cases where the vehicle is equipped with a USBL sensor, a TDOA/ BAOA -based passive navigation scheme was developed ${ }^{[2]}$. A two-stage weighted least squares method for the new TDOA/ BAOA model was proposed ${ }^{[2]}$, in which the time difference and bearing measurements were used. Although it is the latest method designed for the new TDOA/ BAOA model, the solution suffers from the TDOA measurement noise and cannot achieve the CRLB performance. The CRLB is a lower bound on the variance of any unbiased estimator. It tells us the best we can ever expect to be able to do. Thus, for a given localization scenario, the best (smallest) achievable accuracy can be represented by the Cramer-Rao lower bound and many researchers take it as a benchmark for performance evaluation ${ }^{[23][24]}$. Besides, there are some other performance criteria such as Cramer-Rao bound $(\mathrm{CRB})^{[19]}$ or hybrid Bhattacharyya-Barankin (HBB) ${ }^{[20]}$ bound. In this paper, CRLB is used as a benchmark for performance evaluation.

The goal of the paper is to solve the problem in reference [2]. One of that is to derive a new closed-form solution of TDOA/ BAOA -based localization problem in the USBL system, which can achieve the CRLB performance in the case of small measurement noise. The other goal of the paper is that an iterative constrained weighted least squares method based on Taylor expansion is presented to further minimize the localization error in the case of large noise. Although several iteration-based methods were developed based on the closedform solution ${ }^{[23][24][25]}$, the solution will still suffer from the noisy measurements if the noise is large as the time difference and bearing measurements are coupled in the traditional iteration equation. The proposed bias-reduction method can deal with the problem of low iteration accuracy. It can improve the iteration accuracy and reduce the position bias compared with the closed-form solutions used in the traditional TDOA/AOA model, where the azimuth and elevation angles are considered.

The structure of the paper is as follows. The first section is the introduction and includes the current research status and the contributions of the paper. The second section introduces the system models of the source location. The third section introduces the closed-form solution method and the iteration- based method. The fourth section is the analysis of the proposed method. The fifth section verifies the effectiveness of the proposed algorithm through simulation and field tests. The sixth section is the future works. The last section presents summary.

\section{SYSTEM MODEL}

Consider a localization scenario with $M$ basic sensors and their positions are known and denoted by $\boldsymbol{s}_{i}=\left(\begin{array}{lll}x_{i} & y_{i} & z_{i}\end{array}\right)^{T}$ $(i=1,2,3, \ldots M)$. The source position $\left(\boldsymbol{u}_{o}=\left(\begin{array}{lll}x & y & z\end{array}\right)^{T}\right)$ in $N$-dimensional $(N=2$ or 3$)$ space is unknown and needs to be located. The localization scenario is shown in Fig. 1.

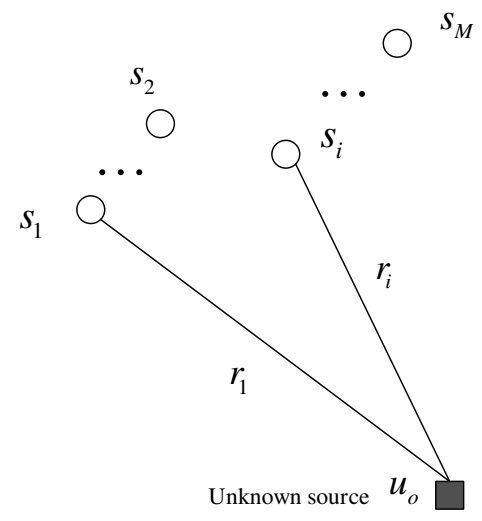

Fig. 1 Source location scenario

We select the first sensor $\left(\boldsymbol{s}_{1}\right)$ as the reference. The sensor array computes the relative time delays of the signals at different sensors with respect to the reference. The TDOA measurement, denoted by $t_{i 1}$, between sensor pairs $i$ and 1 and the corresponding actual range difference of arrival (RDOA) denoted by $r_{i 1}$ are

$$
r_{i 1}=c t_{i 1}+n_{i 1}=r_{i 1}^{0}+n_{i 1}=r_{i}-r_{1}+n_{i 1}
$$

where $r_{i}=\left\|\boldsymbol{u}_{o}-\boldsymbol{s}_{i}\right\|,(i=2,3, \ldots M) . n_{i 1}$ is the measurement noise. Note that $r_{i 1}^{0}$ is actually the range difference which is the TDOA multiplied by the known signal propagation speed c. We shall use time differences and range differences interchangeably throughout the paper as they are differed by a constant scaling factor ${ }^{[26]}$. It is assumed that the TDOA noise vector $\boldsymbol{n}=\left[\begin{array}{lll}n_{21} & \cdots & n_{i 1}\end{array}\right]^{T}$ is zero-mean Gaussian distributed with covariance matrix $\boldsymbol{Q}_{\boldsymbol{r}}=E_{n}\left(\boldsymbol{n} \boldsymbol{n}^{\boldsymbol{T}}\right) . r_{i 1}^{0}$ is the true TDOA measurement.

Take the sensor $\boldsymbol{s}_{i}$ as an example in Fig. 2. The bearing measurements diagram is shown below.

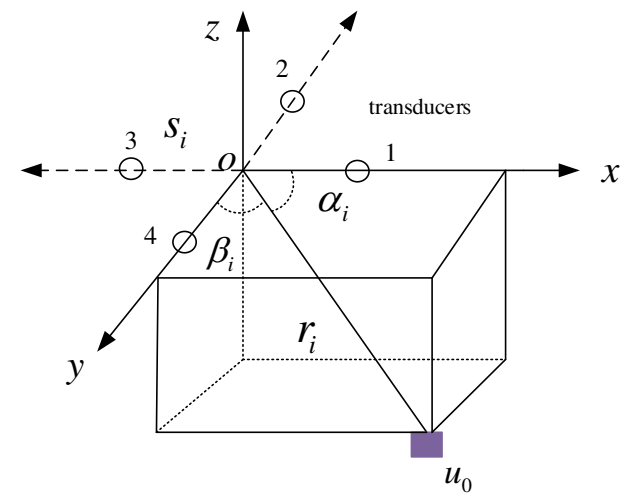

Fig. 2 Bearing information diagram 
The bearing angles can be obtained by the phase difference. Four transducers are installed along the $\mathrm{x}$-axis and $\mathrm{y}$-axis. The transducer array along the $\mathrm{x}$-axis is used to get $\alpha_{i}$ and transducer array along the y-axis is used to get $\beta_{i}$. The phase difference is estimated by the adaptive phase difference estimator, where the Least Mean Square method is used ${ }^{[27]}$.

The bearings are related to the source position and it can be represented as ${ }^{[21]}$.

$$
\left\{\begin{array}{l}
\alpha_{i}=\arccos \left(\frac{x-x_{i}}{r_{i}}\right)+\varepsilon_{i}=\alpha_{i}^{0}+\varepsilon_{i} \\
\beta_{i}=\arccos \left(\frac{y-y_{i}}{r_{i}}\right)+\delta_{i}=\beta_{i}^{0}+\delta_{i}
\end{array}\right.
$$

where $\varepsilon_{i}$ is the measurement noise of bearing $\alpha_{i}$ and $\delta_{i}$ is the measurement noise of bearing $\beta_{i}$. It is assumed that the bearing $\alpha$ noise vector $\boldsymbol{\varepsilon}=\left[\begin{array}{lll}\varepsilon_{1} & \cdots & \varepsilon_{i}\end{array}\right]^{T}$ and bearing $\beta$ noise vector $\boldsymbol{\delta}=\left[\begin{array}{lll}\delta_{1} & \cdots & \delta_{i}\end{array}\right]^{T}$ are zero-mean Gaussian distributed with covariance matrix $\boldsymbol{Q}_{\alpha}=E\left(\boldsymbol{\varepsilon} \boldsymbol{\varepsilon}^{\boldsymbol{T}}\right)$ and $\boldsymbol{Q}_{\boldsymbol{\beta}}=E\left(\boldsymbol{\delta} \boldsymbol{\delta}^{T}\right) . \alpha_{i}^{0}$ and $\beta_{i}^{0}$ are the true bearing information.

\section{PROPOSED METHOD}

In this section, we will develop a closed-form solution method and an iterative constrained bias-reduction method for the system model described in section II.

\section{A. Closed-form solution}

According to the analysis in paper [12], the TDOA model can be simplified as

$$
\begin{gathered}
r_{i 1}^{2}+\left\|\boldsymbol{s}_{1}\right\|^{2}-\left\|\boldsymbol{s}_{i}\right\|^{2}+2\left(\boldsymbol{s}_{i}-\boldsymbol{s}_{1}\right)^{T} \boldsymbol{u}_{o}+2 r_{i 1}\left\|\boldsymbol{u}_{o}-\boldsymbol{s}_{1}\right\| \\
=2\left\|\boldsymbol{u}_{o}-\boldsymbol{s}_{i}\right\| n_{i 1}+n_{i 1}^{2}
\end{gathered}
$$

Taking all the TDOA measurements into consideration, (3) can be extended as follows.

$$
\begin{gathered}
\boldsymbol{h}_{\mathbf{1}}-\boldsymbol{G}_{\mathbf{1}} \boldsymbol{y}=\boldsymbol{B}_{1} \boldsymbol{n}+\boldsymbol{n} \odot \boldsymbol{n} \\
\text { where } \boldsymbol{h}_{\mathbf{1}}=\left[\begin{array}{c}
r_{21}^{2}+\left\|\boldsymbol{s}_{1}\right\|^{2}-\left\|\boldsymbol{s}_{2}\right\|^{2} \\
\vdots \\
r_{M 1}^{2}+\left\|\boldsymbol{s}_{1}\right\|^{2}-\left\|\boldsymbol{s}_{M}\right\|^{2}
\end{array}\right], \\
\boldsymbol{G}_{\mathbf{1}}=-2\left[\begin{array}{cc}
\left(\boldsymbol{s}_{2}-\boldsymbol{s}_{1}\right)^{T} & r_{21} \\
\vdots & \vdots \\
\left(\boldsymbol{s}_{M}-\boldsymbol{s}_{1}\right)^{T} & r_{M 1}
\end{array}\right], \boldsymbol{y}=\left[\begin{array}{ll}
\boldsymbol{u}_{o}^{T} & r_{1}
\end{array}\right]^{T}, \\
\boldsymbol{B}_{1}=2 \operatorname{diag}\left(\left[\begin{array}{ccc}
r_{2} & \ldots & r_{M}
\end{array}\right]\right) .
\end{gathered}
$$

and " $\odot$ " denotes the element-by-element product.

We will derive the pseudo linear equations for the BAOA model. (2) can be reformulated as

$$
\left\{\begin{array}{l}
x-x_{i}=r_{i} \cos \left(\alpha_{i}^{0}\right) \\
y-y_{i}=r_{i} \cos \left(\beta_{i}^{0}\right)
\end{array}\right.
$$

Combine (1) with (5), it can be reformulated as

$$
\left\{\begin{array}{l}
x-x_{i}=\left(r_{i 1}^{0}+r_{1}\right) \cos \left(\alpha_{i}^{0}\right) \\
y-y_{i}=\left(r_{i 1}^{0}+r_{1}\right) \cos \left(\beta_{i}^{0}\right)
\end{array}\right.
$$

In the above equation, $\alpha_{i}^{0}, r_{i 1}^{0}$ and $\beta_{i}^{0}$ are the true values and use their noisy values, (6) becomes

$$
\left\{\begin{array}{l}
x-x_{i}-\left(r_{i 1}+r_{1}\right) \cos \left(\alpha_{i}\right)=\eta_{\alpha} \\
y-y_{i}-\left(r_{i 1}+r_{1}\right) \cos \left(\beta_{i}\right)=\eta_{\beta}
\end{array}\right.
$$

When the measurement noise is small, $\cos \left(\varepsilon_{i}\right) \approx 1$ and $\sin \left(\varepsilon_{i}\right) \approx \varepsilon_{i}$. So we have the following approximation.

$$
\cos \left(\alpha_{i}^{0}+\varepsilon_{i}\right)=\cos \alpha_{i}^{0}-\varepsilon_{i} \sin \alpha_{i}^{0}
$$

Take $x-x_{i}-\left(r_{i 1}+r_{1}\right) \cos \left(\alpha_{i}\right)=\eta_{\alpha}$ as an example. It can be rewritten as.

$$
\begin{aligned}
& x-x_{i}-\left(r_{i 1}+r_{1}\right) \cos \left(\alpha_{i}\right) \\
& =x-x_{i}-\left(r_{i 1}^{0}+n_{i 1}+r_{1}\right) \cos \left(\alpha_{i}^{0}+\varepsilon_{i}\right) \\
& \approx x-x_{i}-\left(r_{i 1}^{0}+r_{1}\right) \cos \left(\alpha_{i}^{0}\right)-\cos \alpha_{i}^{0} n_{i 1}+r_{i} \sin \left(\alpha_{i}^{0}\right) \varepsilon_{i} \\
& \quad \text { Thus, we have }
\end{aligned}
$$

$$
\eta_{\alpha}=r_{i} \sin \left(\alpha_{i}^{0}\right) \varepsilon_{i}-\cos \alpha_{i}^{0} n_{i 1}
$$

The derivation of $\eta_{\beta}$ is the same as $\eta_{\alpha}$ and it can be represented as

$$
\eta_{\beta}=r_{i} \sin \left(\beta_{i}^{0}\right) \delta_{i}-\cos \beta_{i}^{0} n_{i 1}
$$

From (7), (10), and (11), the pseudo linear equations for the BAOA model can be represented as

$$
\text { where } \boldsymbol{h}_{2}=\left[\begin{array}{c}
\boldsymbol{h}_{2}-\boldsymbol{G}_{2} \boldsymbol{y}=\boldsymbol{B}_{2} \boldsymbol{\eta} \\
x_{1} \\
x_{2}+r_{21} \cos \left(\alpha_{2}\right) \\
\vdots \\
x_{M}+r_{M 1} \cos \left(\alpha_{M}\right) \\
y_{1} \\
y_{2}+r_{21} \cos \left(\beta_{2}\right) \\
\vdots \\
y_{M}+r_{M 1} \cos \left(\beta_{M}\right)
\end{array}\right] \text {, }
$$$$
\boldsymbol{G}_{\mathbf{2}}=\left[\begin{array}{cccc}
1 & 0 & 0 & -\cos \left(\alpha_{1}\right) \\
\vdots & \vdots & \vdots & \vdots \\
1 & 0 & 0 & -\cos \left(\alpha_{M}\right) \\
0 & 1 & 0 & -\cos \left(\beta_{1}\right) \\
\vdots & \vdots & \vdots & \vdots \\
0 & 1 & 0 & -\cos \left(\beta_{M}\right)
\end{array}\right], \boldsymbol{B}_{2}=\left[\begin{array}{ccc}
\boldsymbol{B}_{21} & 0_{M \times M} & \boldsymbol{B}_{22} \\
0_{M \times M} & \boldsymbol{B}_{23} & \boldsymbol{B}_{24}
\end{array}\right]
$$

$\boldsymbol{B}_{21}=\operatorname{diag}\left(\left[-r_{1} \sin \left(\alpha_{1}^{0}\right) \quad \ldots \quad-r_{M} \sin \left(\alpha_{M}^{0}\right)\right]\right)$,

$\boldsymbol{B}_{23}=\operatorname{diag}\left(\left[-r_{1} \sin \left(\beta_{1}^{0}\right) \quad \ldots \quad-r_{M} \sin \left(\beta_{M}^{0}\right)\right]\right)$,

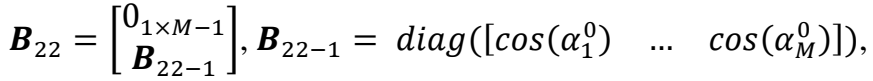

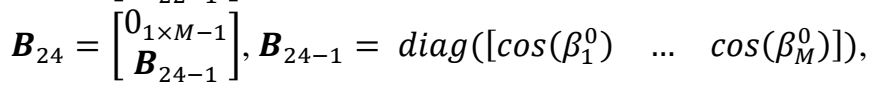
$\eta=\left[\begin{array}{lll}\varepsilon^{T} & \delta^{T} & n^{T}\end{array}\right]^{T}$.

From (4) and (12), the hybrid TDOA/BAOA pseudo linear equation can be represented as

$$
\boldsymbol{h}-\boldsymbol{G} \boldsymbol{y}=\boldsymbol{B} \boldsymbol{\eta}
$$

where $\boldsymbol{h}=\left[\begin{array}{l}\boldsymbol{h}_{\mathbf{2}} \\ \boldsymbol{h}_{\mathbf{1}}\end{array}\right], \boldsymbol{G}=\left[\begin{array}{l}\boldsymbol{G}_{\mathbf{2}} \\ \boldsymbol{G}_{\mathbf{1}}\end{array}\right], \boldsymbol{B}=\left[\begin{array}{cc}\boldsymbol{B}_{2} & \\ 0_{M-1 \times 2 M} & \boldsymbol{B}_{1}\end{array}\right]$.

Applying the weighted least squares method to (13) yields to the solution.

$$
\widetilde{\boldsymbol{y}}=\left(G^{T} W G\right)^{-1} G^{T} W h
$$

where the weighted matrix $\boldsymbol{W}=\left(\boldsymbol{B} \boldsymbol{Q} \boldsymbol{B}^{T}\right)^{-1} \cdot \boldsymbol{Q}=E\left(\boldsymbol{\eta} \boldsymbol{\eta}^{\boldsymbol{T}}\right)$

The estimated $\widetilde{\boldsymbol{y}}$ is as follows.

$$
\widetilde{\boldsymbol{y}}=\left[\begin{array}{ll}
\widetilde{\boldsymbol{u}}_{o}^{T} & {\widetilde{r_{1}}}_{1}
\end{array}\right]^{T}
$$

The estimation error in (15) is defined as

$$
\left\{\begin{array}{l}
\widetilde{\boldsymbol{u}}_{\mathbf{0}}=\boldsymbol{u}_{\mathbf{0}}+\Delta \boldsymbol{u} \\
\widetilde{r_{1}}=r_{1}+\Delta r_{1}
\end{array}\right.
$$

The pseudo linear equation (13) is established under the assumption that the parameters in $\boldsymbol{y}$ are independent. However, $\boldsymbol{u}_{0}$ and $r_{1}$ have the following relationship.

$$
r_{1}=\left\|\boldsymbol{u}_{o}-\boldsymbol{s}_{1}\right\|
$$

Thus, a further step will be carried out to improve the estimation accuracy.

We take the Taylor series expansion of (15) around $\tilde{\boldsymbol{u}}_{\boldsymbol{o}}$ and retaining up to the first-order terms to arrive at

$$
r_{1}=\left\|\boldsymbol{u}_{o}-\boldsymbol{s}_{1}\right\|=\left\|\tilde{\boldsymbol{u}}_{\mathbf{0}}-\boldsymbol{s}_{1}\right\|-\boldsymbol{\rho}_{\widetilde{\boldsymbol{u}}_{0}, \boldsymbol{s}_{1}}^{T} \Delta \boldsymbol{u}
$$

where $\boldsymbol{\rho}_{\widetilde{\mathbf{u}}_{0}, \boldsymbol{s}_{1}}^{T}=\frac{\left(\widetilde{\boldsymbol{u}}_{0}-s_{1}\right)^{T}}{\left\|\widetilde{u}_{0}-s_{1}\right\|}$.

Combing (18) and (16) yields the solution. 


$$
\begin{aligned}
& \qquad \begin{aligned}
\boldsymbol{\epsilon}=\left[\begin{array}{c}
-\Delta \boldsymbol{u} \\
\Delta r_{1}
\end{array}\right] & =\left[\begin{array}{c}
0-\Delta \boldsymbol{u} \\
\widetilde{r}_{1}-\left\|\widetilde{\boldsymbol{u}}_{\mathbf{0}}-\boldsymbol{s}_{1}\right\|+\boldsymbol{\rho}_{\widetilde{\boldsymbol{u}}_{0}, s_{1}}^{T} \Delta \boldsymbol{u}
\end{array}\right] \\
& =\boldsymbol{h}_{3}-\boldsymbol{G}_{3} \Delta \boldsymbol{u} \\
& =\boldsymbol{B}_{3} \Delta \boldsymbol{y}
\end{aligned} \\
& \text { where } \boldsymbol{h}_{\mathbf{3}}=\left[\begin{array}{c}
0_{3 \times 1} \\
\widetilde{r_{1}}-\left\|\widetilde{\boldsymbol{u}}_{0}-\boldsymbol{s}_{1}\right\|
\end{array}\right], \boldsymbol{G}_{3}=\left[\begin{array}{c}
\boldsymbol{I}_{3 \times 3} \\
-\boldsymbol{\rho}_{\widetilde{\boldsymbol{u}}_{0}, s_{1}}^{T}
\end{array}\right],
\end{aligned}
$$$$
\boldsymbol{B}_{3}=\left[\begin{array}{cc}
-\boldsymbol{I}_{3 \times 3} & 0_{3 \times 1} \\
0_{1 \times 3} & 1
\end{array}\right] \text {. }
$$

The estimated error of (14) can be obtained by

$$
\Delta u=\left(G_{3}^{T} W_{3} G_{3}\right)^{-1} G_{3}^{T} W_{3} h_{3}
$$

where $\boldsymbol{W}_{\mathbf{3}}=\left(\boldsymbol{E}\left(\boldsymbol{\epsilon} \boldsymbol{\epsilon}^{\boldsymbol{T}}\right)\right)^{-\mathbf{1}}=\left(\boldsymbol{B}_{3} \operatorname{cov}(y) \boldsymbol{B}_{3}^{T}\right)^{-\mathbf{1}}$

$\operatorname{cov}(y)$ is the covariance matrix of (14). According to the analysis in [28], the covariance matrix can be represented as.

$$
\operatorname{cov}(y)=\left(\boldsymbol{G}^{T} \boldsymbol{W} \boldsymbol{G}\right)^{-1}
$$

The covariance matrix of $\Delta \boldsymbol{u}$ can be shown as ${ }^{[28]}$

$$
\operatorname{cov}(\Delta u)=\left(\boldsymbol{G}_{3}^{T} \boldsymbol{W}_{3} \boldsymbol{G}_{3}\right)^{-1}
$$

Thus, the optimal solution of the source location is

$$
\boldsymbol{u}_{o}=\widetilde{\boldsymbol{u}}_{\mathbf{0}}-\Delta \boldsymbol{u}
$$

B. An improved iterative constrained method based Taylor expansion

Take the Taylor series expansion of $\left\|\boldsymbol{u}_{o}-\boldsymbol{s}_{1}\right\|$ around $\tilde{\boldsymbol{u}}_{o}$ and retaining up to the first-order terms as (18) shows. Substituting (18) and $\widetilde{\boldsymbol{u}}_{o}\left(\widetilde{\boldsymbol{u}}_{o}=\boldsymbol{u}_{o}+\Delta \boldsymbol{u}\right)$ into (3) yields.

$$
\begin{aligned}
r_{i 1}^{2}+\left\|\boldsymbol{s}_{1}\right\|^{2}-\left\|\boldsymbol{s}_{i}\right\|^{2}+2\left(\boldsymbol{s}_{i}-\boldsymbol{s}_{1}\right)^{T}\left(\widetilde{\boldsymbol{u}}_{o}-\Delta \boldsymbol{u}\right) \\
+2 r_{i 1}\left(\left\|\widetilde{\boldsymbol{u}}_{\mathbf{0}}-\boldsymbol{s}_{1}\right\|-\boldsymbol{\rho}_{\widetilde{\boldsymbol{u}}_{0}, \boldsymbol{s}_{1}}^{T} \Delta \boldsymbol{u}\right) \\
=2\left\|\boldsymbol{u}_{o}-\boldsymbol{s}_{i}\right\| n_{i 1}+n_{i 1}^{2}
\end{aligned}
$$

Taking all the TDOA measurements into consideration, (24) can be extended as follows.

where

$$
h_{3}-G_{3} \Delta u=B_{1} n+n \odot n
$$

$\boldsymbol{h}_{3}=$

$\left[\begin{array}{c}r_{21}^{2}+\left\|\boldsymbol{s}_{1}\right\|^{2}-\left\|\boldsymbol{s}_{2}\right\|^{2}+2\left(\boldsymbol{s}_{2}-\boldsymbol{s}_{1}\right)^{T} \widetilde{\boldsymbol{u}}_{o}+2 r_{21}\left\|\widetilde{\boldsymbol{u}}_{\mathbf{0}}-\boldsymbol{s}_{1}\right\| \\ \vdots \\ r_{i 1}^{2}+\left\|\boldsymbol{s}_{1}\right\|^{2}-\left\|\boldsymbol{s}_{i}\right\|^{2}+2\left(\boldsymbol{s}_{i}-\boldsymbol{s}_{1}\right)^{T} \widetilde{\boldsymbol{u}}_{o}+2 r_{i 1}\left\|\widetilde{\boldsymbol{u}}_{\mathbf{0}}-\boldsymbol{s}_{1}\right\|\end{array}\right]$,

$\boldsymbol{G}_{3}=2\left[\begin{array}{c}\left(\boldsymbol{s}_{2}-\boldsymbol{s}_{1}\right)^{T}+r_{21} \boldsymbol{\rho}_{\widetilde{\boldsymbol{u}}_{0}, \boldsymbol{s}_{1}}^{T} \\ \vdots \\ \left(\boldsymbol{s}_{i}-\boldsymbol{s}_{1}\right)^{T}+r_{i 1} \boldsymbol{\rho}_{\widetilde{\boldsymbol{u}}_{0}, \boldsymbol{s}_{1}}^{T}\end{array}\right]$.

Similarly, substituting (18) into (7) yields.

$$
\begin{gathered}
\tilde{x}-\Delta x-x_{i}-\left(r_{i 1}+\left\|\widetilde{\boldsymbol{u}}_{\mathbf{0}}-\boldsymbol{s}_{1}\right\|-\boldsymbol{\rho}_{\widetilde{\boldsymbol{u}}_{\mathbf{0}}, \boldsymbol{s}_{1}}^{T} \Delta \boldsymbol{u}\right) \cos \left(\alpha_{i}\right) \\
=\eta_{\alpha} \\
\tilde{y}-\Delta y-y_{i}-\left(r_{i 1}+\left\|\widetilde{\boldsymbol{u}}_{\mathbf{0}}-\boldsymbol{s}_{1}\right\|-\boldsymbol{\rho}_{\widetilde{\boldsymbol{u}}_{\mathbf{0}}, \boldsymbol{s}_{\mathbf{1}}}^{T} \Delta \boldsymbol{u}\right) \cos \left(\beta_{i}\right) \\
=\eta_{\beta}
\end{gathered}
$$

Taking all the BAOA measurements into consideration, (26) can be extended as follows.

$$
\text { where } \boldsymbol{h}_{\mathbf{4}}=\left[\begin{array}{c}
\boldsymbol{h}_{\mathbf{4}}-\boldsymbol{G}_{\mathbf{4}} \Delta \boldsymbol{u}=\boldsymbol{B}_{2} \boldsymbol{\eta} \\
x_{1}-\tilde{x}+\cos \left(\alpha_{1}\right)\left\|\widetilde{\boldsymbol{u}}_{\mathbf{0}}-\boldsymbol{s}_{1}\right\| \\
x_{2}-\tilde{x}+r_{21} \cos \left(\alpha_{2}\right)+\cos \left(\alpha_{2}\right)\left\|\widetilde{\boldsymbol{u}}_{\mathbf{0}}-\boldsymbol{s}_{1}\right\| \\
\vdots \\
x_{M}-\tilde{x}+r_{M 1} \cos \left(\alpha_{M}\right)+\cos \left(\alpha_{M}\right)\left\|\widetilde{\boldsymbol{u}}_{\mathbf{0}}-\boldsymbol{s}_{1}\right\| \\
y_{1}-\tilde{y}+\cos \left(\beta_{1}\right)\left\|\tilde{\boldsymbol{u}}_{\mathbf{0}}-\boldsymbol{s}_{1}\right\| \\
y_{2}-\tilde{y}+r_{21} \cos \left(\beta_{2}\right)+\cos \left(\beta_{2}\right)\left\|\widetilde{\boldsymbol{u}}_{\mathbf{0}}-\boldsymbol{s}_{1}\right\| \\
\vdots \\
y_{M}-\tilde{y}+r_{M 1} \cos \left(\beta_{M}\right)+\cos \left(\beta_{M}\right)\left\|\tilde{\boldsymbol{u}}_{\mathbf{0}}-\boldsymbol{s}_{1}\right\|
\end{array}\right],
$$

$\boldsymbol{G}_{\mathbf{4}}=\left[\begin{array}{ccc}\cos \left(\alpha_{1}\right) \rho_{x}-1 & \cos \left(\alpha_{1}\right) \rho_{y} & \cos \left(\alpha_{1}\right) \rho_{z} \\ \vdots & \vdots & \vdots \\ \cos \left(\alpha_{M}\right) \rho_{x}-1 & \cos \left(\alpha_{M}\right) \rho_{y} & \cos \left(\alpha_{M}\right) \rho_{z} \\ \cos \left(\beta_{1}\right) \rho_{x} & \cos \left(\beta_{1}\right) \rho_{y}-1 & \cos \left(\beta_{1}\right) \rho_{z} \\ \vdots & \vdots & \vdots \\ \cos \left(\beta_{M}\right) \rho_{x} & \cos \left(\beta_{M}\right) \rho_{y}-1 & \cos \left(\beta_{M}\right) \rho_{z}\end{array}\right]$. $\boldsymbol{\rho}_{\widetilde{\boldsymbol{u}}_{\mathbf{0}}, \boldsymbol{s}_{1}}^{T}=\left[\begin{array}{lll}\rho_{x} & \rho_{y} & \rho_{z}\end{array}\right]$.

From (25) and (27), the hybrid TDOA/BAOA equation can be represented as

$$
\boldsymbol{h}-\boldsymbol{G} \Delta \boldsymbol{u}=\boldsymbol{B \eta}
$$

where $\boldsymbol{h}=\left[\begin{array}{l}\boldsymbol{h}_{\mathbf{2}} \\ \boldsymbol{h}_{\mathbf{1}}\end{array}\right], \boldsymbol{G}=\left[\begin{array}{l}\boldsymbol{G}_{\mathbf{2}} \\ \boldsymbol{G}_{\mathbf{1}}\end{array}\right], \boldsymbol{B}=\left[\begin{array}{rr}\boldsymbol{B}_{2} & \\ 0_{M-1 \times 2 M} & \boldsymbol{B}_{1}\end{array}\right]$.

Applying the iteration method to (28) yields to the solution.

$$
\begin{gathered}
\widetilde{\boldsymbol{u}}_{0}^{k+1}=\widetilde{\boldsymbol{u}}_{0}^{k}-\Delta \boldsymbol{u} \\
=\widetilde{\boldsymbol{u}}_{0}^{k}+\left(G^{T} W G\right)^{-1} G^{T} W h
\end{gathered}
$$

where $k$ represents the $\mathrm{k} t h$ iteration.

However, the solution will suffer from the noisy measurement. For example, the TDOA measurements are coupled in $\boldsymbol{h}_{\mathbf{4}}$ and the estimated results cannot attain the CRLB performance if the TDOA noise is large. Thus, we will further consider the noisy measurement in the coefficient matrix of the iteration method.

We expand the parameters and coefficient matrix as follows.

$$
\begin{gathered}
\boldsymbol{A}=\left[\begin{array}{ll}
-\boldsymbol{G} & \boldsymbol{h}
\end{array}\right] \\
\boldsymbol{V}=\left[\begin{array}{ll}
\Delta \boldsymbol{u}^{\boldsymbol{T}} & 1
\end{array}\right]^{T}
\end{gathered}
$$

Define the cost function with the expanded parameters as follows.

$$
J=\min (A V)^{T} W A V
$$

Since $\boldsymbol{A}$ contains the measurement noise and it can be decomposed as.

$$
\boldsymbol{A}=\Delta \boldsymbol{A}+\boldsymbol{A}^{0}
$$

where $\boldsymbol{A}^{0}$ is a matrix without any measurement noise. $\Delta \boldsymbol{A}=$ $\left[\begin{array}{ll}-\Delta \boldsymbol{G} & \boldsymbol{\Delta} \boldsymbol{h}\end{array}\right]$ is the noise term, which can be expressed as follows.

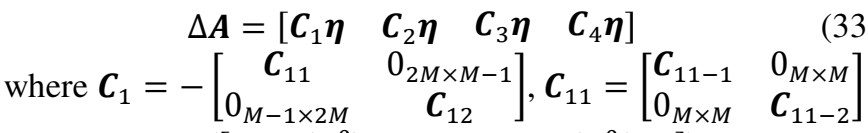
$\boldsymbol{C}_{11-1}=\operatorname{diag}\left(\left[\begin{array}{lll}-\sin \left(\alpha_{1}^{0}\right) \rho_{x} & \ldots & -\sin \left(\alpha_{M}^{0}\right) \rho_{x}\end{array}\right]\right)$,

$\boldsymbol{C}_{11-2}=\operatorname{diag}\left(\left[-\sin \left(\beta_{1}^{0}\right) \rho_{x} \quad \ldots \quad-\sin \left(\beta_{M}^{0}\right) \rho_{x}\right]\right)$,

$\boldsymbol{C}_{12}=\operatorname{diag}(\underbrace{\left[\begin{array}{ccc}\rho_{x} & \cdots & \rho_{x}\end{array}\right]}_{M-1})$,

$\boldsymbol{C}_{2}=\left[\begin{array}{cc}\boldsymbol{C}_{21} & 0_{2 M \times M-1} \\ 0_{M-1 \times 2 M} & \boldsymbol{C}_{22}\end{array}\right], \boldsymbol{C}_{21}=\left[\begin{array}{cc}\boldsymbol{C}_{21-1} & 0_{M \times M} \\ 0_{M \times M} & \boldsymbol{C}_{21-2}\end{array}\right]$,

$\boldsymbol{C}_{21-1}=\operatorname{diag}\left(\left[\begin{array}{lll}-\sin \left(\alpha_{1}^{0}\right) \rho_{y} & \ldots & -\sin \left(\alpha_{M}^{0}\right) \rho_{y}\end{array}\right]\right)$,

$\boldsymbol{C}_{21-2}=\operatorname{diag}\left(\left[\begin{array}{lll}-\sin \left(\beta_{1}^{0}\right) \rho_{y} & \ldots & -\sin \left(\beta_{M}^{0}\right) \rho_{y}\end{array}\right]\right)$,

$\boldsymbol{C}_{22}=\operatorname{diag}(\underbrace{\left[\begin{array}{ccc}\rho_{y} & \cdots & \rho_{y}\end{array}\right]}_{M-1})$,

$\boldsymbol{C}_{3}=\left[\begin{array}{cc}\boldsymbol{C}_{31} & 0_{2 M \times M-1} \\ 0_{M-1 \times 2 M} & \boldsymbol{C}_{32}\end{array}\right], \boldsymbol{C}_{31}=\left[\begin{array}{ll}\boldsymbol{C}_{31-1} & 0_{M \times M} \\ 0_{M \times M} & \boldsymbol{C}_{31-2}\end{array}\right]$,

$\boldsymbol{C}_{31-1}=\operatorname{diag}\left(\left[\begin{array}{lll}-\sin \left(\alpha_{1}^{0}\right) \rho_{z} & \ldots & -\sin \left(\alpha_{M}^{0}\right) \rho_{z}\end{array}\right]\right)$,

$\boldsymbol{C}_{31-2}=\operatorname{diag}\left(\left[-\sin \left(\beta_{1}^{0}\right) \rho_{z} \quad \ldots \quad-\sin \left(\beta_{M}^{0}\right) \rho_{z}\right]\right)$,

$\boldsymbol{C}_{32}=\operatorname{diag}(\underbrace{\left[\begin{array}{ccc}\rho_{z} & \cdots & \rho_{z}\end{array}\right]}_{M-1})$,

$\boldsymbol{C}_{4}=\left[\begin{array}{cc}\boldsymbol{C}_{41} & \boldsymbol{C}_{42} \\ 0_{M-1 \times 2 M} & \boldsymbol{C}_{43}\end{array}\right], \boldsymbol{C}_{41}=\left[\begin{array}{ll}\boldsymbol{C}_{41-1} & 0_{M \times M} \\ 0_{M \times M} & \boldsymbol{C}_{41-2}\end{array}\right]$, 


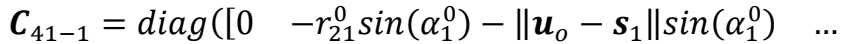

$$
\begin{aligned}
& \left.\left.-r_{M 1}^{0} \sin \left(\alpha_{M}^{0}\right)-\left\|\boldsymbol{u}_{o}-\boldsymbol{s}_{1}\right\| \sin \left(\alpha_{M}^{0}\right)\right]\right)
\end{aligned}
$$

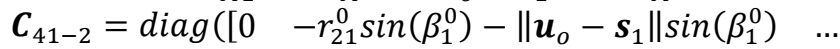

$$
\begin{aligned}
& \left.\left.-r_{M 1}^{0} \sin \left(\beta_{M}^{0}\right)-\left\|\boldsymbol{u}_{o}-\boldsymbol{s}_{1}\right\| \sin \left(\beta_{M}^{0}\right)\right]\right) \\
& \boldsymbol{C}_{42}=\left[\begin{array}{ccc}
0_{1 \times M} & \\
\operatorname{diag}\left(\left[\cos \left(\alpha_{1}^{0}\right)\right.\right. & \ldots & \left.\left.\left.\cos \left(\alpha_{M}^{0}\right)\right]\right)\right) \\
0_{1 \times M} & \\
\operatorname{diag}\left(\left[\cos \left(\beta_{1}^{0}\right)\right.\right. & \ldots & \left.\left.\cos \left(\beta_{M}^{0}\right)\right]\right)
\end{array}\right] \text {, } \\
& \boldsymbol{C}_{43}=2 \operatorname{diag}\left(\left[\begin{array}{lll}
r_{21}^{0} & \ldots & r_{M 1}^{0}
\end{array}\right]\right) \\
& +2 \operatorname{diag}\left(\left[\left\|\boldsymbol{u}_{o}-\boldsymbol{s}_{1}\right\| \quad \ldots \quad\left\|\boldsymbol{u}_{o}-\boldsymbol{s}_{1}\right\|\right]\right) .
\end{aligned}
$$

Substituting (32) into (31) yields the cost function

$$
J=\boldsymbol{V}^{T} \boldsymbol{A}^{0^{T}} \boldsymbol{W} \boldsymbol{A}^{0} \boldsymbol{V}+\boldsymbol{V}^{T} \Delta \boldsymbol{A}^{T} \boldsymbol{W} \Delta \boldsymbol{A} \boldsymbol{V}+2 \boldsymbol{V}^{T} \Delta \boldsymbol{A}^{T} \boldsymbol{W} \boldsymbol{A}^{0} \boldsymbol{V}
$$

If we take the expectation of $J$ and the third term $\boldsymbol{V}^{T} \Delta \boldsymbol{A}^{T} \boldsymbol{W} \boldsymbol{A}^{0} \boldsymbol{V}$ will vanish due to the fact that $\Delta A$ is zero-mean. The expectation of $J$ can be obtained.

$$
E(J)=\boldsymbol{V}^{T} A^{0^{T}} \boldsymbol{W} \boldsymbol{A}^{0} \boldsymbol{V}+\boldsymbol{V}^{T} E\left[\Delta \boldsymbol{A}^{T} \boldsymbol{W} \Delta \boldsymbol{A}\right] \boldsymbol{V}
$$

In (30), we treat the second term as a constant constraint to (31). Thus, the cost function can be reformulated as

$$
\begin{gathered}
\min \boldsymbol{V}^{T} \boldsymbol{A}^{T} \boldsymbol{W} \boldsymbol{A} \boldsymbol{V} \\
\text { s.t. } \boldsymbol{V}^{T} \boldsymbol{\Omega} \boldsymbol{V}=k
\end{gathered}
$$

where $\boldsymbol{\Omega}=E\left[\Delta \boldsymbol{A}^{T} \boldsymbol{W} \Delta \boldsymbol{A}\right]$ and the constant k can be any value.

$$
\begin{gathered}
\boldsymbol{\Omega}=E\left[\Delta \boldsymbol{A}^{T} \boldsymbol{W} \Delta \boldsymbol{A}\right] \\
=\left[\begin{array}{llll}
\boldsymbol{\Omega}_{\mathbf{1 1}} & \boldsymbol{\Omega}_{\mathbf{1 2}} & \boldsymbol{\Omega}_{\mathbf{1 3}} & \boldsymbol{\Omega}_{\mathbf{1 4}} \\
\boldsymbol{\Omega}_{\mathbf{2 1}} & \boldsymbol{\Omega}_{22} & \boldsymbol{\Omega}_{23} & \boldsymbol{\Omega}_{\mathbf{2 4}} \\
\boldsymbol{\Omega}_{\mathbf{3 1}} & \boldsymbol{\Omega}_{32} & \boldsymbol{\Omega}_{33} & \boldsymbol{\Omega}_{\mathbf{3 4}} \\
\boldsymbol{\Omega}_{\mathbf{4 1}} & \boldsymbol{\Omega}_{\mathbf{4 2}} & \boldsymbol{\Omega}_{\mathbf{4 3}} & \boldsymbol{\Omega}_{\mathbf{4 4}}
\end{array}\right]
\end{gathered}
$$

where $\boldsymbol{\Omega}_{\mathbf{1 1}}=\operatorname{tr}\left(\boldsymbol{C}_{\mathbf{1}} \boldsymbol{W} \boldsymbol{C}_{\mathbf{1}}^{T} \boldsymbol{Q}\right), \mathbf{\Omega}_{\mathbf{1 2}}=\operatorname{tr}\left(\boldsymbol{C}_{\mathbf{1}} \boldsymbol{W} \boldsymbol{C}_{\mathbf{2}}^{T} \boldsymbol{Q}\right)$,

$\boldsymbol{\Omega}_{\mathbf{1 3}}=\operatorname{tr}\left(\boldsymbol{C}_{1} \boldsymbol{W} \boldsymbol{C}_{\mathbf{3}}^{T} \boldsymbol{Q}\right), \boldsymbol{\Omega}_{\mathbf{1 4}}=\operatorname{tr}\left(\boldsymbol{C}_{1} \boldsymbol{W} \boldsymbol{C}_{\mathbf{4}}^{T} \boldsymbol{Q}\right), \boldsymbol{\Omega}_{\mathbf{2 1}}=\boldsymbol{\Omega}_{\mathbf{1 2}}^{T}$,

$\boldsymbol{\Omega}_{\mathbf{2 2}}=\operatorname{tr}\left(\boldsymbol{C}_{2} \boldsymbol{W} \boldsymbol{C}_{\mathbf{2}}^{T} \boldsymbol{Q}\right), \boldsymbol{\Omega}_{\mathbf{2 3}}=\operatorname{tr}\left(\boldsymbol{C}_{2} \boldsymbol{W} \boldsymbol{C}_{3}^{T} \boldsymbol{Q}\right)$,

$\boldsymbol{\Omega}_{\mathbf{2 4}}=\operatorname{tr}\left(\boldsymbol{C}_{2} \boldsymbol{W} \boldsymbol{C}_{\mathbf{4}}^{T} \boldsymbol{Q}\right), \boldsymbol{\Omega}_{\mathbf{3 1}}=\boldsymbol{\Omega}_{\mathbf{1 3}}^{T}, \boldsymbol{\Omega}_{\mathbf{3 2}}=\boldsymbol{\Omega}_{\mathbf{2 3}}^{T}$,

$\boldsymbol{\Omega}_{\mathbf{3}}=\operatorname{tr}\left(\boldsymbol{C}_{3} \boldsymbol{W} \boldsymbol{C}_{3}^{T} \boldsymbol{Q}\right), \boldsymbol{\Omega}_{\mathbf{3 4}}=\operatorname{tr}\left(\boldsymbol{C}_{3} \boldsymbol{W} \boldsymbol{C}_{\mathbf{4}}^{T} \boldsymbol{Q}\right), \boldsymbol{\Omega}_{\mathbf{4 1}}=\boldsymbol{\Omega}_{\mathbf{1 4}}^{T}$,

$\boldsymbol{\Omega}_{\mathbf{4 4}}=\operatorname{tr}\left(\boldsymbol{C}_{4} \boldsymbol{W} \boldsymbol{C}_{4}^{T} \boldsymbol{Q}\right), \boldsymbol{\Omega}_{\mathbf{4 2}}=\boldsymbol{\Omega}_{\mathbf{2 4}}^{T}, \boldsymbol{\Omega}_{\mathbf{4 3}}=\boldsymbol{\Omega}_{\mathbf{3 4}}^{T}$.

The Lagrange multiplier can be used to solve the constrained minimization problem. Combine the Lagrange multiplier with (36) and it can be reformulated as.

$$
\min \boldsymbol{V}^{T} \boldsymbol{A}^{T} \boldsymbol{W} \boldsymbol{A} \boldsymbol{V}-\boldsymbol{\lambda}\left(k-\boldsymbol{V}^{T} \boldsymbol{\Omega} \boldsymbol{V}\right)
$$

Taking the derivative with respect to $\boldsymbol{V}$ and setting it to zero yield

$$
\left(\boldsymbol{A}^{T} \boldsymbol{W} \boldsymbol{A}\right) \boldsymbol{V}=\boldsymbol{\lambda} \boldsymbol{\Omega} \boldsymbol{V}
$$

According to the generalized singular value decomposition (GSVD) theory, the solution $\boldsymbol{V}$ is the eigenvector of the pair $\left(\begin{array}{ll}\boldsymbol{A}^{T} \boldsymbol{W} \boldsymbol{A} & \boldsymbol{\Omega}\end{array}\right)$ which corresponds to the minimum generalized eigenvalue.

The estimated $\Delta \boldsymbol{u}$ can be obtained by.

$$
\Delta \boldsymbol{u}=\frac{\boldsymbol{V}(1: 3)}{\boldsymbol{V}(\mathbf{4})}
$$

Thus, the whole algorithm can be summarized as follows.

\footnotetext{
Algorithm 1: A TDOA/BAOA-based localization method based on iterative constrained weighted least squares

Step 1. Set $\boldsymbol{B}=\boldsymbol{I}_{\mathbf{3 M - 1}}$ and solve the WLS problem (14). A rough value $\widetilde{\boldsymbol{y}}$ can be obtained.

Step 2. Reformulate the weighting matrix $\mathbf{W}$ with the rough value $\widetilde{\boldsymbol{y}}$ and get the closed-form of the TDOA/BAOA localization problem using (14) and (23).
}

Step 3. Initialize $k=0$. Define a convergence threshold $\varepsilon$, a divergence threshold $\sigma$, and a maximum number of iterations $\tau$. Set the initial value $\widetilde{\boldsymbol{u}}^{\mathbf{0}}$ with the closed-form solution in step 2 .

Step 4. Set $k=k+1$. Formulate the constraint WLS optimization problem (31) with $\widetilde{\boldsymbol{u}}^{k}$.

Step 5. Solve the constraint WLS optimization (31) based on GSVD to obtain the estimated value $\Delta \boldsymbol{u}$.

Step 6. Obtain the optimal value $\widetilde{\boldsymbol{u}}^{\boldsymbol{k}+1}=\widetilde{\boldsymbol{u}}^{\boldsymbol{k}}-\Delta \boldsymbol{u}$ and reformulate the weighting matrix $\mathbf{W}$ with $\widetilde{\boldsymbol{u}}^{\boldsymbol{k}+\mathbf{1}}$

Step 7. If $\Delta \boldsymbol{u}<\varepsilon$ or k $>\tau$, go to step 8 , otherwise, go to step 4 . Step 8. $u_{o}=\widetilde{u}^{k+1}$.

End

The above iteration algorithm is based on the closed-form solution in section III.A. In the case of small noise, the closedform solution is very close to the CRLB performance. Usually, two or three iterations are sufficient to ensure the convergence.

\section{CRLB AND PERFORMANCE ANALYSIS}

\section{A. CRLB for TDOA/BAOA}

The logarithmic probability density function of the bearing information vector is.

$$
\ln \left(\boldsymbol{m} ; \boldsymbol{u}_{\mathbf{0}}\right)=k-\frac{1}{2}\left(\boldsymbol{m}-\boldsymbol{m}^{\boldsymbol{o}}\right)^{T} Q_{m}^{-1}\left(\boldsymbol{m}-\boldsymbol{m}^{\boldsymbol{o}}\right)
$$

where $\boldsymbol{m}=[\boldsymbol{\alpha} ; \boldsymbol{\beta}]$ is the actual bearing information with noise and $\boldsymbol{m}^{\boldsymbol{o}}=\left[\boldsymbol{\alpha}^{\boldsymbol{o}} ; \boldsymbol{\beta}^{\boldsymbol{o}}\right]$ is the real bearing. $Q_{m}$ is block diagonal with diagonal blocks $\boldsymbol{Q}_{\boldsymbol{\alpha}}$ and $\boldsymbol{Q}_{\boldsymbol{\beta}} \cdot k$ is a constant which is independent with the measurement ${ }^{[29]} \cdot \alpha=\left[\begin{array}{lll}\alpha_{1} & \ldots & \alpha_{M}\end{array}\right]^{T}$,

$$
\boldsymbol{\alpha}^{o}=\left[\begin{array}{lll}
\alpha_{1}^{o} & \ldots & \alpha_{M}^{o}
\end{array}\right]^{T}, \boldsymbol{\beta}=\left[\begin{array}{lll}
\beta_{1} & \ldots & \beta_{M}
\end{array}\right]^{T},
$$$$
\boldsymbol{\beta}^{o}=\left[\begin{array}{lll}
\beta_{1}^{o} & \ldots & \beta_{M}^{o}
\end{array}\right]^{T} \text {. }
$$

The CRLB matrix of $\boldsymbol{u}_{\mathbf{0}}$ with bearing measurement is

$$
\operatorname{CRLB}_{A O A}\left(\boldsymbol{u}_{\mathbf{0}}\right)=F I M^{-1}\left(\boldsymbol{u}_{\mathbf{0}}\right)
$$

where FIM is the Fisher information matrix and it can be represented as.

$$
F I M\left(\boldsymbol{u}_{\mathbf{0}}\right)=\left(\frac{\partial \boldsymbol{m}^{\boldsymbol{o}}}{\partial \boldsymbol{u}_{\mathbf{0}}}\right)^{T} Q_{m}^{-1}\left(\frac{\partial \boldsymbol{m}^{\boldsymbol{o}}}{\partial \boldsymbol{u}_{\mathbf{0}}}\right)
$$

The detailed derivation of $\frac{\partial \boldsymbol{m}^{\boldsymbol{o}}}{\partial \boldsymbol{u}_{\mathbf{0}}}$ can be found in Appendix A.

The CRLB for the hybrid TDOA/BAOA measurement can be represented as.

$$
\mathrm{CRLB}_{T D O A / A O A}\left(\boldsymbol{u}_{\mathbf{0}}\right)=\left[\left(\frac{\partial \boldsymbol{t}^{\boldsymbol{o}}}{\partial \boldsymbol{u}_{\mathbf{0}}}\right)^{T} Q^{-1}\left(\frac{\partial \boldsymbol{t}^{\boldsymbol{o}}}{\partial \boldsymbol{u}_{\mathbf{0}}}\right)\right]^{-1}
$$

where $\boldsymbol{t}=[\boldsymbol{\alpha} ; \boldsymbol{\beta} ; \boldsymbol{R}]$ is the actual bearing and TDOA information with noise and $\boldsymbol{t}^{\boldsymbol{o}}=\left[\boldsymbol{\alpha}^{\boldsymbol{o}} ; \boldsymbol{\beta}^{\boldsymbol{o}} ; \boldsymbol{R}^{\boldsymbol{o}}\right]$ is the real value without noise. $\boldsymbol{Q}=E\left(\boldsymbol{\eta} \boldsymbol{\eta}^{\boldsymbol{T}}\right) . \boldsymbol{R}=\left[\begin{array}{lll}r_{21} & \ldots & r_{M 1}\end{array}\right]^{\boldsymbol{T}}, \boldsymbol{R}^{\boldsymbol{o}}=$ $\left[\begin{array}{lll}r_{21}^{O} & \ldots & r_{M 1}^{O}\end{array}\right]^{T}$. $\frac{\partial t^{o}}{\partial u_{0}}$ can be expressed as follows.

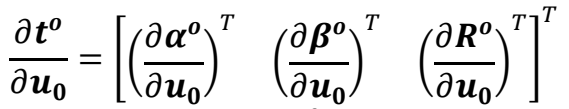

The detailed derivation of $\frac{\partial R^{o}}{\partial u_{0}}$ can be found in [12]. Thus, the $\mathrm{CRLB}_{\text {TDOA/AOA }}$ can be obtained.

\section{B. Algorithm performance}

One of the contributions of the paper is to derive the closed- 
form solution using the time difference and bearing measurements in the USBL system.

We will compare the estimation covariance with the CRLB to establish the approximate efficiency of the closed-form solution.

From (20), (21), and (22), the inverse of the estimated covariance matrix can be expressed as.

$$
\begin{aligned}
(\operatorname{cov}(\Delta \boldsymbol{u}))^{-1} & =\boldsymbol{G}_{3}^{T} \boldsymbol{W}_{3} \boldsymbol{G}_{\mathbf{3}} \\
& =\boldsymbol{G}_{3}^{T}\left(\boldsymbol{B}_{3} \operatorname{cov}(y) \boldsymbol{B}_{3}^{T}\right)^{-1} \boldsymbol{G}_{3} \\
& =\boldsymbol{G}_{3}^{T}\left(\boldsymbol{B}_{3}\left(\boldsymbol{G}^{\boldsymbol{T}}\left(\boldsymbol{B} \boldsymbol{Q} \boldsymbol{B}^{T}\right)^{-1} \boldsymbol{G}\right)^{-1} \boldsymbol{B}_{3}^{T}\right)^{-1} \boldsymbol{G}_{3} \\
& =\boldsymbol{G}_{3}^{T}\left(\boldsymbol{B}_{3}^{T}\right)^{-1} \boldsymbol{G}^{T}\left(\boldsymbol{B}^{T}\right)^{-1} \boldsymbol{Q}^{-1} \boldsymbol{B}^{-1} \boldsymbol{G} \boldsymbol{B}_{3}^{-1} \boldsymbol{G}_{3} \\
& =G_{4}^{T} \boldsymbol{Q}^{-1} G_{4}
\end{aligned}
$$

where $G_{4}=\boldsymbol{B}^{-1} \boldsymbol{G} \boldsymbol{B}_{3}^{-1} \boldsymbol{G}_{3}$.

Compare (46) with the $\left(\mathrm{CRLB}_{T D O A / A O A}\right)^{-1}$ and it shows that they have the same functional form. To prove the performance of the closed-form solution, we just need to prove that $G_{4} \approx$ $\left(\frac{\partial t^{o}}{\partial u_{0}}\right)$ holds.

$G_{4}$ can be expressed as follows.

$$
G_{4}=\left[\begin{array}{l}
G_{41} \\
G_{42}
\end{array}\right]
$$

The detailed derivation of $G_{41}$ and $G_{42}$ can be found in Appendix B.

According to the analysis in [28], $G_{42}$ can be proved to have the following relationship.

$$
G_{42}=M_{2}^{-1} \boldsymbol{G}_{\mathbf{1}} \boldsymbol{B}_{3}^{-1} \boldsymbol{G}_{3}=-\frac{\partial \boldsymbol{R}^{\boldsymbol{o}}}{\partial \boldsymbol{u}_{\mathbf{0}}}
$$

Take the measurement vector $\boldsymbol{\alpha}$ as an example.

Consider the following relationship.

$$
r_{1} \sin \left(\alpha_{1}^{o}\right)=\sqrt{r_{1}^{2}-r_{1}^{2} \cos ^{2}\left(\alpha_{1}^{o}\right)}=\sqrt{r_{1}^{2}-\left(x-x_{1}\right)^{2}}
$$

Put (49) and the definitions of $\boldsymbol{\rho}_{\tilde{u}_{0}, s_{1}}^{T}$ into $G_{41}$. The first row of $G_{41}$ can be expressed as follows.

$$
\begin{aligned}
& G_{41}(1,1)=\frac{1}{\left\|u_{0}-s_{1}\right\| \sin \left(\alpha_{1}^{o}\right)}-\frac{\rho_{\tilde{u}_{0}, s_{1}}^{T}(x) \cos \left(\alpha_{1}^{o}\right)}{\left\|u_{0}-s_{1}\right\| \sin \left(\alpha_{1}^{o}\right)} \\
& =\frac{1}{\sqrt{1-\left(\frac{x-x_{1}}{\left\|u_{0}-s_{1}\right\|}\right)^{2}}}\left[\frac{1}{\left\|u_{0}-s_{1}\right\|}-\frac{\left(x-x_{i}\right)^{2}}{\left(\left(u_{0}-s_{i}\right)^{T}\left(u_{0}-s_{i}\right)\right)^{1.5}}\right] \\
& G_{41}(1,2)=-\frac{\rho_{\tilde{u}_{0}, s_{1}}^{T}(y) \cos \left(\alpha_{1}^{o}\right)}{\left\|u_{0}-s_{1}\right\| \sin \left(\alpha_{1}^{o}\right)}= \\
& \frac{1}{\sqrt{1-\left(\frac{x-x_{1}}{\left\|u_{0}-s_{1}\right\|}\right)^{2}}}\left(-\frac{\left(x-x_{1}\right)\left(\mathrm{y}-y_{1}\right)}{\left(\left(u_{0}-s_{1}\right)^{T}\left(u_{0}-s_{1}\right)\right)^{1.5}}\right) \\
& G_{41}(1,3)=-\frac{\rho_{\widetilde{u}_{0}, s_{1}}^{T}(z) \cos \left(\alpha_{1}^{o}\right)}{\left\|u_{0}-s_{1}\right\| \sin \left(\alpha_{1}^{o}\right)}= \\
& \frac{1}{\sqrt{1-\left(\frac{x-x_{1}}{\left\|u_{0}-s_{1}\right\|}\right)^{2}}}\left(-\frac{\left(x-x_{1}\right)\left(\mathrm{z}-z_{1}\right)}{\left(\left(u_{0}-s_{1}\right)^{T}\left(u_{0}-s_{1}\right)\right)^{1.5}}\right)
\end{aligned}
$$

According to the analysis in Appendix C, the $i$ th rows of $G_{41}$ can be expressed as follows. $(i=2, \ldots M)$.

$$
G_{41}(i, 1)=\frac{1}{\sqrt{1-\left(\frac{x-x_{i}}{r_{i}}\right)^{2}}}\left[\frac{1}{r_{i}}-\frac{\left(x-x_{i}\right)^{2}}{r_{i}^{3}}\right]
$$

$$
\begin{aligned}
& G_{41}(i, 2)=\frac{1}{\sqrt{1-\left(\frac{x-x_{i}}{r_{i}}\right)^{2}}}\left[-\frac{\left(x-x_{i}\right)\left(y-y_{i}\right)}{r_{i}^{3}}\right] \\
& G_{41}(i, 3)=\frac{1}{\sqrt{1-\left(\frac{x-x_{i}}{r_{i}}\right)^{2}}}\left[-\frac{\left(x-x_{i}\right)\left(z-z_{i}\right)}{r_{i}^{3}}\right]
\end{aligned}
$$

The $j$ th rows of $G_{41}$ have the same functional form as the $i$ th rows. $(i=2, \ldots M),(j=M+1, \ldots 2 M)$.Thus, the $j$ th rows of $G_{41}$ can be expressed as follows.

$$
\begin{gathered}
G_{41}(j, 1)=\frac{1}{\sqrt{1-\left(\frac{y-y_{i}}{r_{i}}\right)^{2}}}\left[-\frac{\left(x-x_{i}\right)\left(y-y_{i}\right)}{r_{i}^{3}}\right] \\
G_{41}(j, 2)=\frac{1}{\sqrt{1-\left(\frac{y-y_{i}}{r_{i}}\right)^{2}}}\left[\frac{1}{r_{i}}-\frac{\left(y-y_{i}\right)^{2}}{r_{i}^{3}}\right] \\
G_{41}(j, 3)=\frac{1}{\sqrt{1-\left(\frac{y-y_{i}}{r_{i}}\right)^{2}}}\left[-\frac{\left(z-z_{i}\right)\left(y-y_{i}\right)}{r_{i}^{3}}\right]
\end{gathered}
$$

From (50)-(52), it can be obtained.

$$
G_{41}=-\frac{\partial \boldsymbol{m}^{o}}{\partial \boldsymbol{u}_{0}}
$$

According to (48) and (53), it can be obtained.

$$
G_{4}=\left[\begin{array}{c}
-\frac{\partial \boldsymbol{m}^{o}}{\partial \boldsymbol{u}_{0}} \\
-\frac{\partial \boldsymbol{R}^{o}}{\partial \boldsymbol{u}_{0}}
\end{array}\right]=\left(\frac{\partial \boldsymbol{t}^{o}}{\partial \boldsymbol{u}_{\mathbf{0}}}\right)
$$

Thus, the estimation covariance can be expressed as follows.

$$
(\operatorname{cov}(\Delta \boldsymbol{u}))^{-1} \approx \mathrm{CRLB}_{T D O A / A O A}
$$

This completes the proof that the proposed closed-form solution can achieve the CRLB accuracy.

\section{SIMULATION AND FIELD TEST ANALYSIS}

\section{A. simulation test}

To verify the effectiveness of the proposed algorithm, simulations are performed in this section. Several tests are carried out and 1000 Monte Carlo simulations are performed for each test.

Symbols used for the simulations are as follows:

1) 'TDOA' denotes that only the time difference measurement is used for the source localization and the positioning method is described in [12].

2) 'BAOA' denotes only the bearing information is used for the source localization and the positioning method is the Gauss-Newton iteration method with the true value. The number of iterations is set to 2 .

3) 'T-TDOA/BAOA' denotes the existing method for the source localization with the time difference and bearing information, which is described in [2].

4) 'ML' denotes the maximum likelihood estimation method based on Gauss-Newton iteration as (29) shows.

5) 'Proposed method' denotes the proposed method described in section III. The number of iterations is set to 4 .

The root mean square error (RMSE) and the bias norm of the source position is used to evaluate the localization accuracy. They are defined as follows. 


$$
\begin{gathered}
\text { RMSE }=\sqrt{\frac{\sum_{i=1}^{L}\left\|\widehat{\boldsymbol{u}}_{o}-\boldsymbol{u}_{o}\right\|^{2}}{L}} \\
\text { BiasNorm }=\left\|\frac{\sum_{i=1}^{L} \widehat{\boldsymbol{u}}_{o}}{L}-\boldsymbol{u}_{o}\right\|
\end{gathered}
$$

where $\boldsymbol{u}_{o}$ denotes the true source position. $\mathrm{L}=50000$ is the number of ensemble runs.

The TDOA/BAOA noise is Gaussian and the covariance matrix is modeled as

$$
\begin{gathered}
\boldsymbol{Q}_{\boldsymbol{m}}=\operatorname{diag}\left(\begin{array}{ll}
\sigma_{s}^{2} \boldsymbol{I}_{M} & \left.\sigma_{s}^{2} \boldsymbol{I}_{M}\right) \\
\boldsymbol{Q}_{\boldsymbol{r}}=\sigma_{d}^{2}\left(\boldsymbol{I}_{M-1}+\mathbf{1}_{M-1} \mathbf{1}_{M-1}^{T}\right) / 2
\end{array}\right.
\end{gathered}
$$

The sensor positions are given by

$$
s_{i}=\left[50 \cos \left(\frac{\pi}{4}(i-2)\right) \quad 50 \sin \left(\frac{\pi}{4}(i-2)\right) \quad z_{i}\right]^{T}
$$

where $i=2, \ldots . \mathrm{M}$ is the sensor number. $z_{i}$ is generated randomly between $(-4,4)$.

The reference sensor is located at $s_{1}=\left[\begin{array}{lll}0 & 0 & 0\end{array}\right]^{T}$. The sensors are distributed uniformly in a circle. Two different source locations are selected in the following simulation. One is outside of the circle formed by the sensors and $u_{0}$ is chosen randomly as $u_{0}=\left[\begin{array}{lll}150 & 65 & -30\end{array}\right]^{T}$. And the other is inside of the circle formed by the sensors. Thus, $u_{0}$ is chosen randomly as $u_{0}=\left[\begin{array}{lll}20 & 40 & -30\end{array}\right]^{T}$.

\section{Test 1-The impact of the TDOA noise on Localization \\ Accuracy}

In this scenario, $\sigma_{s}^{2}$ is a constant value $\left(\sigma_{s}^{2}=0.001 \mathrm{rad}^{2}\right)$, $\mathrm{M}=7$.

When the source is located inside of the circle formed by the sensors, compare the RMSE and the bias norm of the estimated source position with different methods.

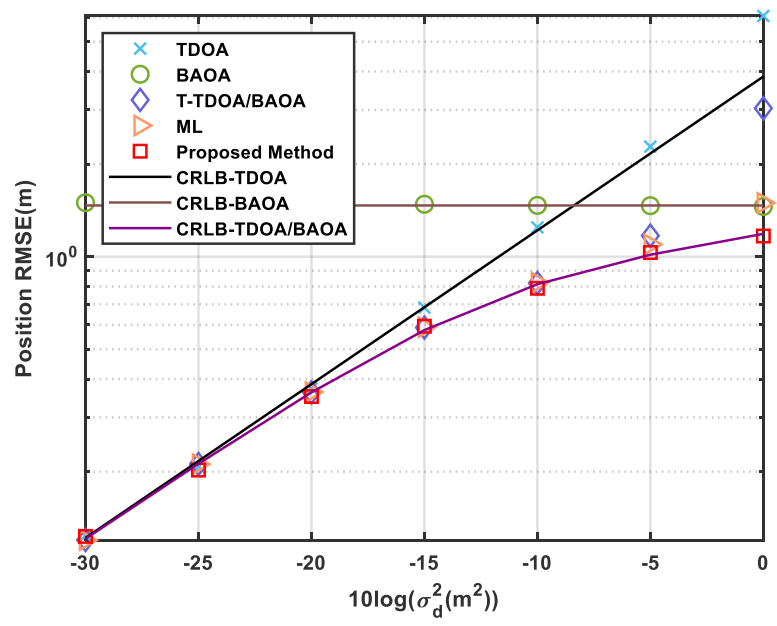

Fig. 3 Comparison of RMSE with different methods. $\left(u_{0}=\right.$ $\left.\left[\begin{array}{lll}20 & 40 & -30\end{array}\right]^{T}\right)$

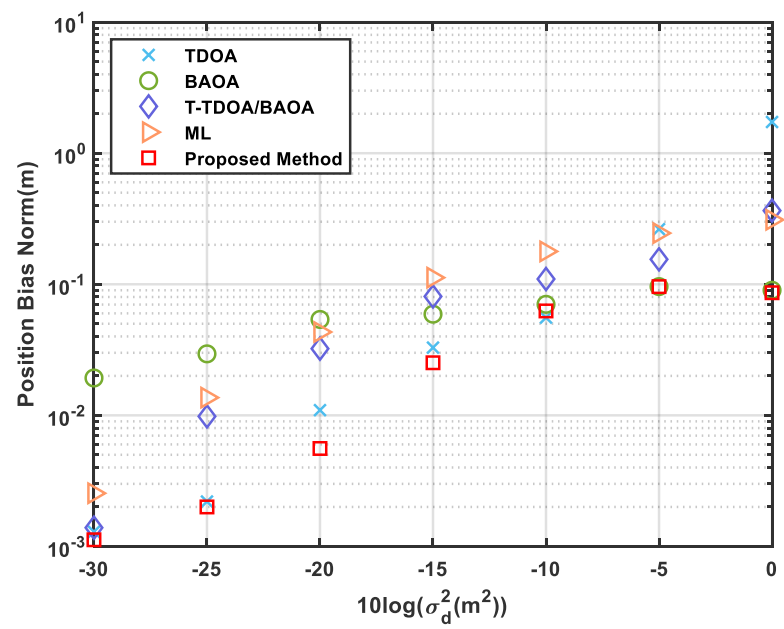

Fig. 4 Comparison of bias norm with different methods. $\left(u_{0}=\right.$ $\left[\begin{array}{lll}20 & 40 & -30\end{array}\right]^{T}$ )

When the source is located outside of the circle formed by the sensors, compare the RMSE and the bias norm of the estimated source position with different methods.

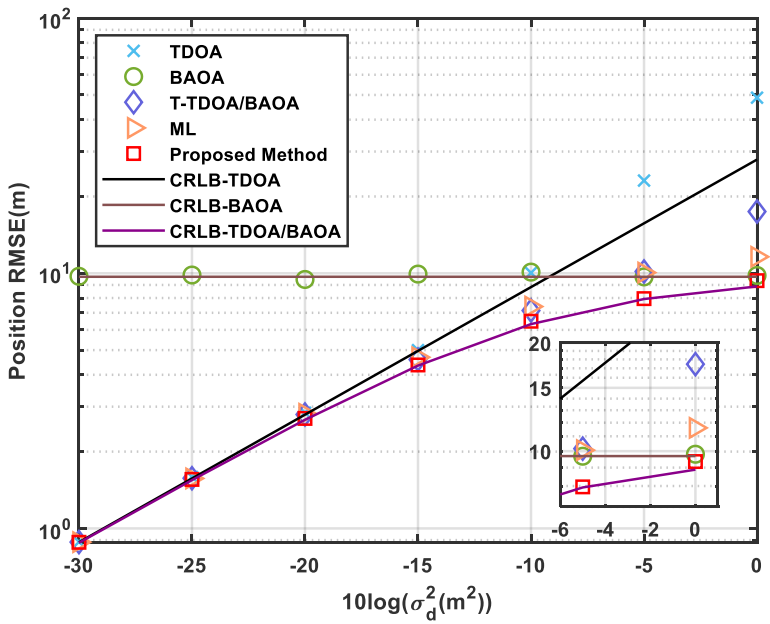

Fig. 5 Comparison of RMSE with different methods. $\left(u_{0}=\right.$ $\left[\begin{array}{lll}150 & 65 & -30\end{array}\right]^{T}$ )

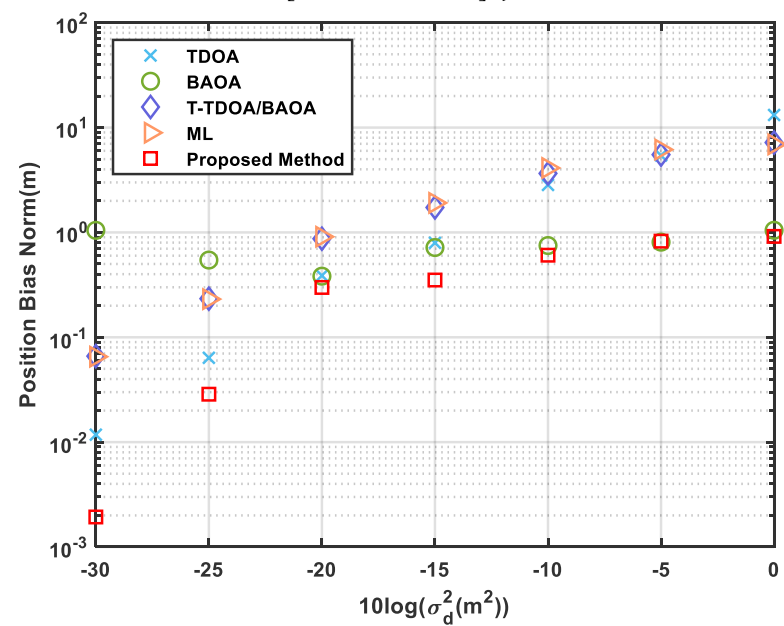

Fig. 6 Comparison of bias norm with different methods. $\left(u_{0}=\right.$ $\left.\left[\begin{array}{lll}150 & 65 & -30\end{array}\right]^{T}\right)$

Fig. 3 and Fig. 5 show the estimation accuracy of the proposed method is higher than that of using only TDOA or BAOA. The performance superiority of the hybrid TDOA/BAOA CRLB is still obvious than that of using TDOA 
only or BAOA only. Compared with the T-TDOA/BAOA method, the proposed method achieves the CRLB accuracy better. The T-TDOA/BAOA method can only achieve the CRLB accuracy in the case of small noise. When the TDOA noise is large, the estimation accuracy of the T-TDOA/BAOA method will decrease. Due to the noisy measurements, the traditional iteration method cannot attain the CRLB performance with the noise increases. However, the proposed method still has the best estimation accuracy. Take the scenario where the source is inside of the circle formed by the sensors as an example. When $\sigma_{d}^{2}=1 \mathrm{~m}^{2}$, that is to say the following results would correspond to a range error of 1 meter, the RMSE of the proposed method is $1.16 \mathrm{~m}$, which is smaller than the TTDOA/BAOA $(3.04 \mathrm{~m})$ and $\mathrm{ML}(1.50 \mathrm{~m})$. It has a $1.88-\mathrm{m}$ reduction in RMSE as compared with the T-TDOA/BAOA and a 0.34-m reduction in RMSE as compared with the ML. Take the scenario where the source is outside of the circle formed by the sensors as an example. When $\sigma_{d}^{2}=1 \mathrm{~m}^{2}$, that is to say the following results would correspond to a range error of 1 meter, the RMSE of the proposed method is $9.35 \mathrm{~m}$, which is smaller than the T-TDOA/BAOA (17.46m) and ML (11.62m). It has an 8.11-m reduction in RMSE as compared with the TTDOA/BAOA and a 2.27-m reduction in RMSE as compared with the ML.

It can be seen from both Fig. 3 and Fig. 5 that the values for the proposed method are sometimes below the CRLB, e.g. when $u_{0}=\left[\begin{array}{lll}20 & 40 & -30\end{array}\right]^{T}$, the value is below CRLB at a range error of 1 meter $\left(\sigma_{d}^{2}=1 \mathrm{~m}^{2}\right)$. It can be attributed to the fact that the method is biased compared to an unbiased CRLB.

Fig. 4 and Fig. 6 show the bias norm of the source position with different methods. It's obvious that the bias norm of the proposed method is the smallest among these methods. Take the scenario where the source is inside of the circle formed by the sensors as an example. When $\sigma_{d}^{2}=1 \mathrm{~m}^{2}$, the bias norm of the proposed method is $0.08 \mathrm{~m}$, which is smaller than the TTDOA/BAOA $(0.36 \mathrm{~m})$ and ML $(0.31 \mathrm{~m})$. It has a $0.28-\mathrm{m}$ reduction in bias norm as compared with the T-TDOA/BAOA and a $0.23-\mathrm{m}$ reduction in bias norm as compared with the ML. Take the scenario where the source is outside of the circle formed by the sensors as an example. When $\sigma_{d}^{2}=1 \mathrm{~m}^{2}$, the bias norm of the proposed method is $0.91 \mathrm{~m}$, which is smaller than the T-TDOA/BAOA $(7.20 \mathrm{~m})$ and ML $(6.91 \mathrm{~m})$. It has a $6.29-\mathrm{m}$ reduction in bias norm as compared with the T-TDOA/BAOA and a $6.0-\mathrm{m}$ reduction in bias norm as compared with the ML. Although the proposed method has an only $2-m$ reduction in RMSE compared with the ML method, the localization bias is greatly reduced.

This completes the analysis that the proposed method has a higher position accuracy and smaller estimation bias when the TDOA noise changes. It achieves the CRLB accuracy well even when the TDOA noise is large.

\section{Test 2-The impact of BAOA noise on Localization Accuracy}

In this scenario, $\sigma_{d}^{2}$ is a constant value $\left(\sigma_{d}^{2}=0.3162 m^{2}\right)$, $\mathrm{M}=7$.
When the source is located inside of the circle formed by the sensors, compare the RMSE and the bias norm of the estimated source position with different methods.

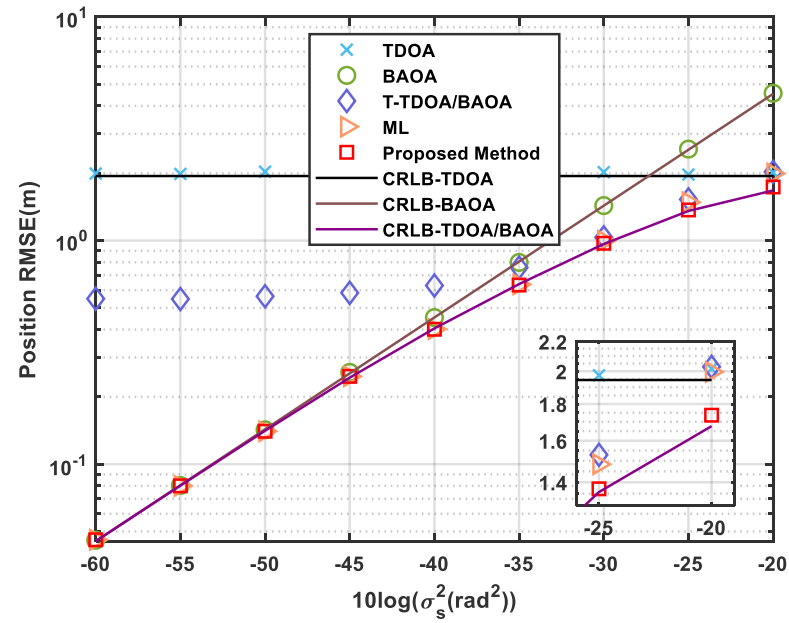

Fig. 7 Comparison of RMSE with different methods. $\left(u_{0}=\right.$ $\left.\left[\begin{array}{lll}20 & 40 & -30\end{array}\right]^{T}\right)$

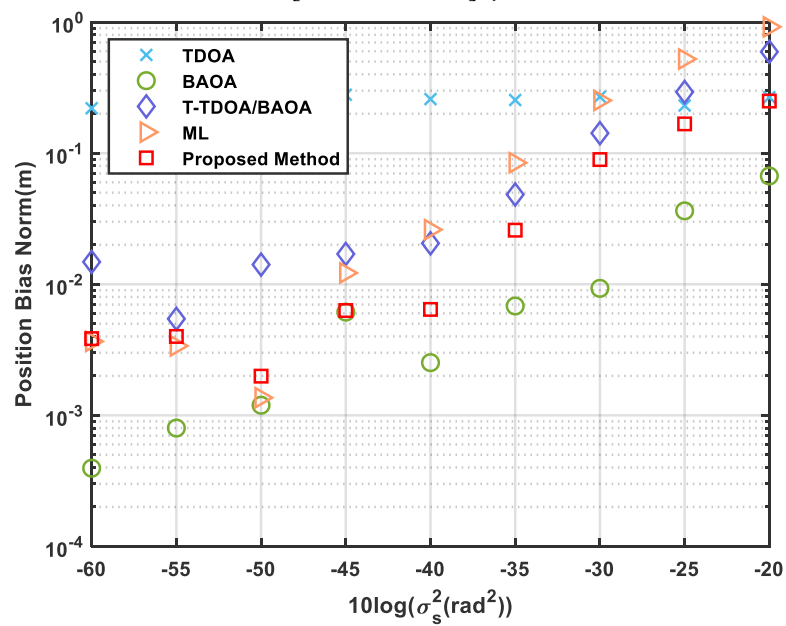

Fig. 8 Comparison of bias norm with different methods. $\left(u_{0}=\right.$ $\left[\begin{array}{lll}20 & 40 & -30\end{array}\right]^{T}$ )

When the source is located inside of the circle formed by the sensors, compare the RMSE and the bias norm of the estimated source position with different methods.

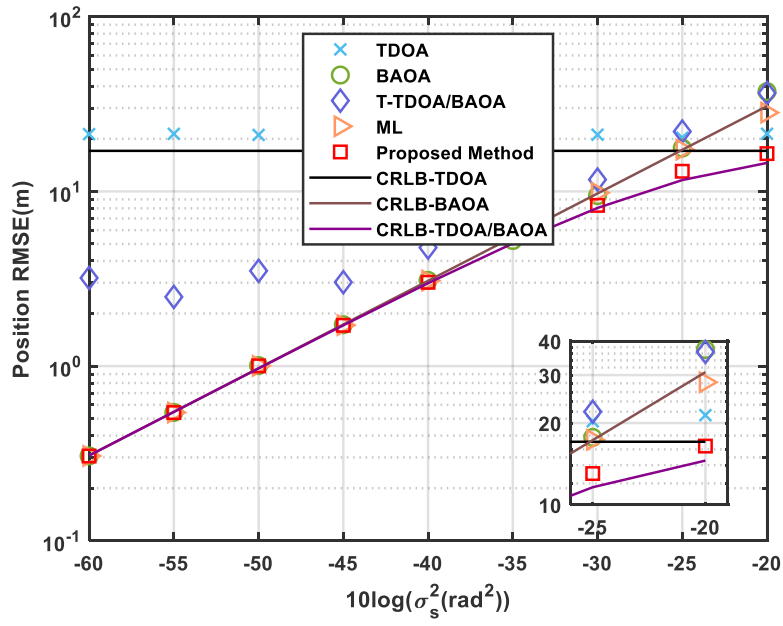

Fig. 9 Comparison of RMSE with different methods.. $\left(u_{0}=\right.$ $\left[\begin{array}{lll}150 & 65 & -30\end{array}\right]^{T}$ ) 


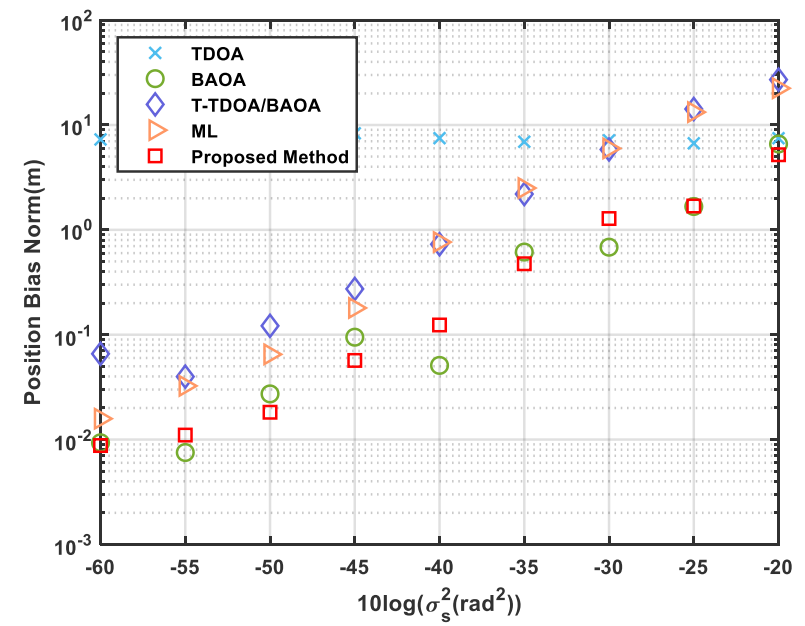

Fig. 10 Comparison of bias norm with different methods. $\left(u_{0}=\right.$ $\left.\left[\begin{array}{lll}150 & 65 & -30\end{array}\right]^{T}\right)$

Fig. 7 and Fig. 9 show the estimation accuracy of the proposed method is higher than that of using only TDOA or BAOA. The performance superiority of the hybrid CRLB of TDOA/BAOA is still obvious than that of using TDOA only or BAOA only. Compared with the T-TDOA/BAOA method, the proposed method achieves the CRLB accuracy better. When the BAOA noise is small, the T-TDOA/BAOA method still cannot achieve the CRLB accuracy. It ignores the influence of the TDOA measurement noise in the noise coefficient matrix $\boldsymbol{B}_{2}$. Thus, the TDOA measurement will affect the estimation accuracy more even if the BAOA noise is small. The proposed method and the ML method can achieve the CRLB accuracy in the case of small BAOA noise. With the increase of the BAOA noise, the proposed method has the best estimation accuracy. Take the scenario where the source is inside of the circle formed by the sensors as an example. When $\sigma_{s}^{2}=0.0032 \mathrm{rad}^{2}$, that is to say the following results would correspond to a range error of 3.24 degree. the RMSE of the proposed method is $1.37 \mathrm{~m}$, which is smaller than the T-TDOA/BAOA (1.53m) and ML (1.48m). It has a $0.16-\mathrm{m}$ reduction in RMSE as compared with the T-TDOA/BAOA and a $0.11-\mathrm{m}$ reduction in RMSE as compared with the ML. Take the scenario where the source is outside of the circle formed by the sensors as an example. When $\sigma_{s}^{2}=0.0032 \mathrm{rad}^{2}$, the RMSE of the proposed method is $13.04 \mathrm{~m}$, which is smaller than the T-TDOA/BAOA $(22.00 \mathrm{~m})$ and ML (17.34m). It has an 8.96-m reduction in RMSE as compared with the T-TDOA/BAOA and a 4.3-m reduction in RMSE as compared with the ML. Thus, the proposed method can attain the CRLB performance no matter the BAOA noise is small or large.

Fig. 8 and Fig. 10 show the bias norm of the source position with different methods. It's obvious that the bias norm of the proposed method is smaller than the T-TDOA/BAOA and ML methods, which further proves that the proposed method cannot only improve the estimation accuracy but also reduce the position bias. However, the bias norm from BAOA-based localization model is better than the results from the TDOA/BAOA-based localization model (the proposed method and the T-TDOA/BAOA method). This is because the bias is caused by the nonlinearity issue and the noisy measurement ${ }^{[30]}$.
In the case of the same BAOA noise, large TDOA noise in the TDOA/BAOA localization model will lead to greater positioning bias than BAOA model. Take the scenario where the source is inside of the circle formed by the sensors as an example. When $\sigma_{s}^{2}=0.0032 \mathrm{rad}^{2}$, the bias norm of the proposed method is $0.16 \mathrm{~m}$, which is smaller than the TTDOA/BAOA $(0.29 \mathrm{~m})$ and $\mathrm{ML}(0.52 \mathrm{~m})$. It has a $0.13-\mathrm{m}$ reduction in bias norm as compared with the T-TDOA/BAOA and a $0.36-\mathrm{m}$ reduction in bias norm as compared with the ML. Take the scenario where the source is outside of the circle formed by the sensors as an example. When $\sigma_{s}^{2}=0.0032 \mathrm{rad}^{2}$, the bias norm of the proposed method is $1.36 \mathrm{~m}$, which is smaller than the T-TDOA/BAOA (14.20m) and ML (13.27m). It has a 12.84-m reduction in bias norm as compared with the T-TDOA/BAOA and a 11.91-m reduction in bias norm as compared with the ML.

This completes the analysis that the proposed method has a higher position accuracy and smaller estimation bias when the BAOA noise changes. It achieves the CRLB accuracy well even when the BAOA noise is large.

\section{Scenario 3-The impact of the sensor numbers}

In this scenario, $\sigma_{d}^{2}$ is a constant value $\left(\sigma_{d}^{2}=0.3162 \mathrm{~m}^{2}\right)$ and $\sigma_{s}^{2}$ is a constant value $\left(\sigma_{s}^{2}=0.0032 \mathrm{rad}^{2}\right)$. The sensor number is varied from 6 to 10 .

Take the scenario where the source is outside of the circle formed by the sensors as an example. Compare the RMSE and the bias norm of the estimated source position with different methods.

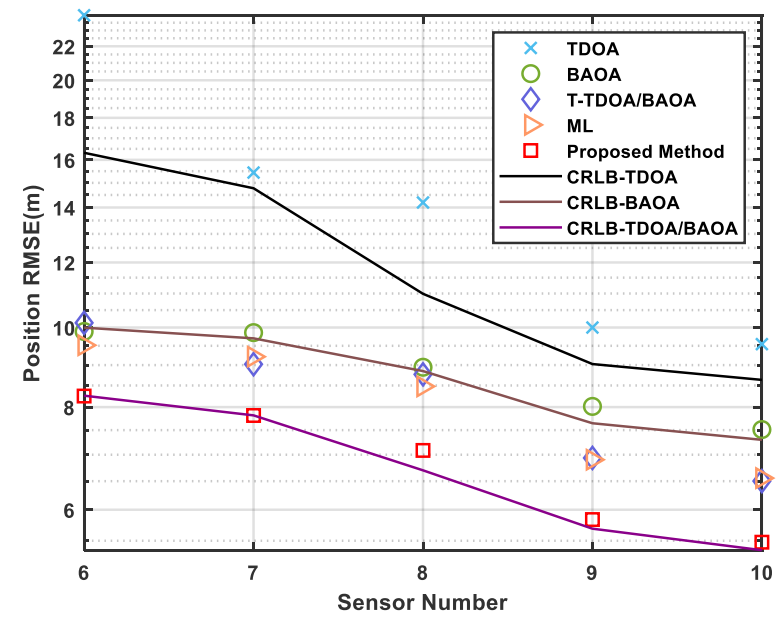

Fig. 11 Comparison of RMSE with different methods. 


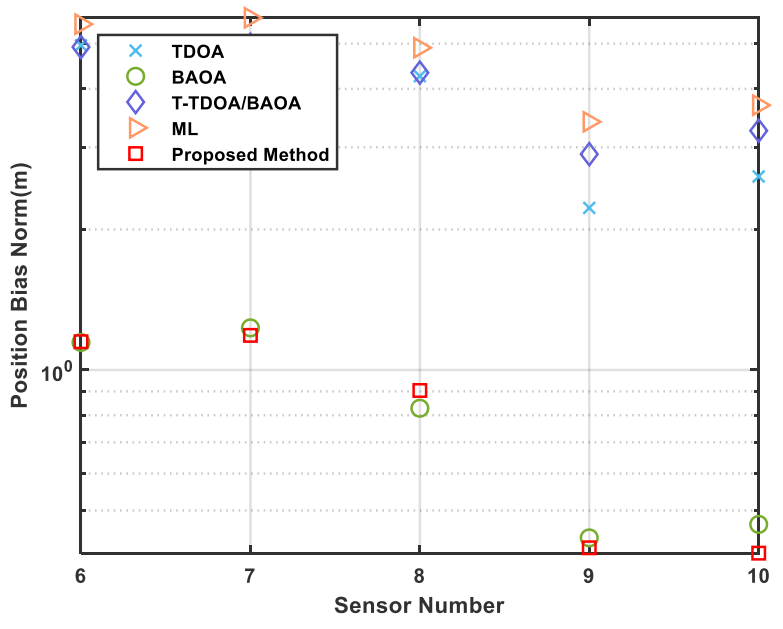

Fig. 12 Comparison of bias norm with different methods.

Fig. 11 shows the proposed method has the best estimation performance no matter how the sensor number changes, e.g., when the sensor number is 10, the RMSE of the proposed method is $5.48 \mathrm{~m}$, which is smaller than the T-TDOA/BAOA $(6.50 \mathrm{~m})$ and $\mathrm{ML}(6.56 \mathrm{~m})$. It has a $1.02-\mathrm{m}$ reduction in RMSE as compared with the T-TDOA/BAOA and a $1.08-\mathrm{m}$ reduction in RMSE as compared with the ML. And the estimation accuracy is increased with the increase of the sensor number.

Fig. 12 shows the bias norm of the source position with different methods. It's obvious that the bias norm of the proposed method is the smallest among these methods. e.g., when the sensor number is 10 , the bias norm of the proposed method is $0.40 \mathrm{~m}$, which is smaller than the T-TDOA/BAOA (3.26m) and $\mathrm{ML}(3.69 \mathrm{~m})$. It has a 2.86-m reduction in bias norm as compared with the T-TDOA/BAOA and a 3.29-m reduction in bias norm as compared with the ML.

This completes the analysis that the proposed method has a higher position accuracy and smaller estimation bias when the sensor number changes.

The average computation times, using MATLAB in a personal computer with core (TM) i5-4460, and the main frequency is $3.2 \mathrm{GHz}$ are listed in Table I.

TABLE I

AVERAGE COMPUTATION TIMES

\begin{tabular}{lcclll}
\hline algorithms & TDOA & BAOA & $\begin{array}{l}\text { T- } \\
\text { TDOA/BAOA }\end{array}$ & ML & $\begin{array}{l}\text { Proposed } \\
\text { method }\end{array}$ \\
\hline $\begin{array}{l}\text { Average } \\
\text { time (s) }\end{array}$ & 0.004 & 0.013 & 0.009 & 0.025 & 0.034 \\
\hline
\end{tabular}

It shows that the computational cost of the traditional methods such as the TDOA and the T-TDOA/BAOA is less than that of the proposed method and the ML method. Although the average computation time of the proposed method is larger, it has a better estimation performance. In the engineering aspect, if the algorithms are implemented on some chips with high configurations, which will meet the requirements of the computational efficiency, the proposed method is a suitable choice.

\section{B. Field test}

To verify the effectiveness of the proposed algorithm, the TDOA/BAOA-based localization problem will be verified with the field test data in this section.
As described in the paper [2], the USBL can provide time difference and bearing measurements and USBL can be applied to the underwater TDOA/BAOA-based localization problem. Thus, the USBL data is utilized to verify the effectiveness of the TDOA/BAOA algorithm.

The transponder is placed on the underwater equipment and the transceiver array is equipped on the vessel. The schematic diagram of USBL is as follows.

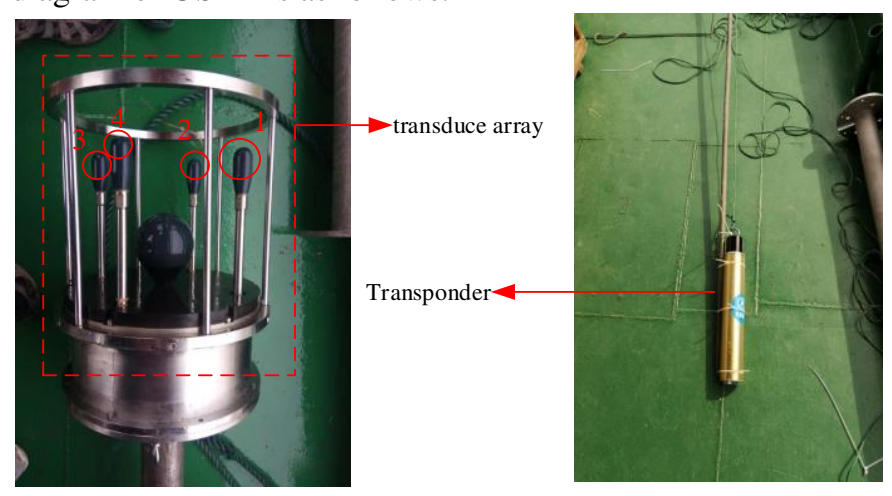

Fig. 13 Schematic diagram of USBL

The four transducers are distributed along the $\mathrm{x}$-axis and $\mathrm{y}-$ axis, which are marked with a red circle. The distribution of transducer array is the same as that in Fig. 2. Bearing $\alpha$ is obtained by the phase difference of transducer 1 and 3 and Bearing $\beta$ is obtained by the phase difference of transducer 2 and 4.

The transponder is treated as a source with an unknown position. The ship is equipped with transceiver array voyages around the source. The ship position is measured by RTK GPS and the accuracy of RTK can reach the level of a centimeter. The TDOA/BAOA-based localization problem is treated as the underwater source localization problem with the known ship position and the USBL data, which is shown below.

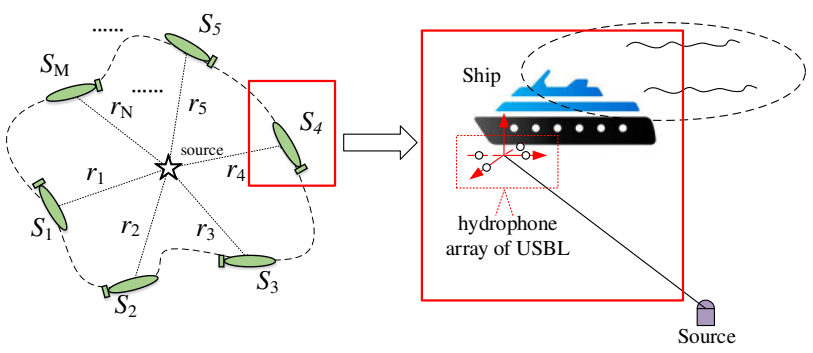

Fig. 14 Simplified underwater source localization problem

The true position of the transponder is calibrated by the long baseline method, which is the author's previous work. The detailed calibration process can be found in [31]. As the accuracy of the long-baseline method reaches the level of a centimeter, it can be used as the truth value of algorithm verification. The altitude coordinate of the transponder is $-9 \mathrm{~m}$. The altitude coordinates of the USBL position are around $9.5 \mathrm{~m}$.

The underwater experiment was carried on the Yangtze River in Nanjing city. With the available USBL data and the ship position, the positioning results of different algorithms will be compared.

\section{Test 1}

The ship's trajectory is a circle and it moves slowly around the source. The trajectory is marked as the black line in Fig. 15. 
Several ship positions are selected as the reference to calculate the source, which is marked as a blue circle. In this test, the source is inside of the circle formed by the sensors. Take the first ship position as the reference position and show their positions in rectangular coordinates as the right picture in Fig. 15 shows. The true source position is marked as a red square, which is shown below.
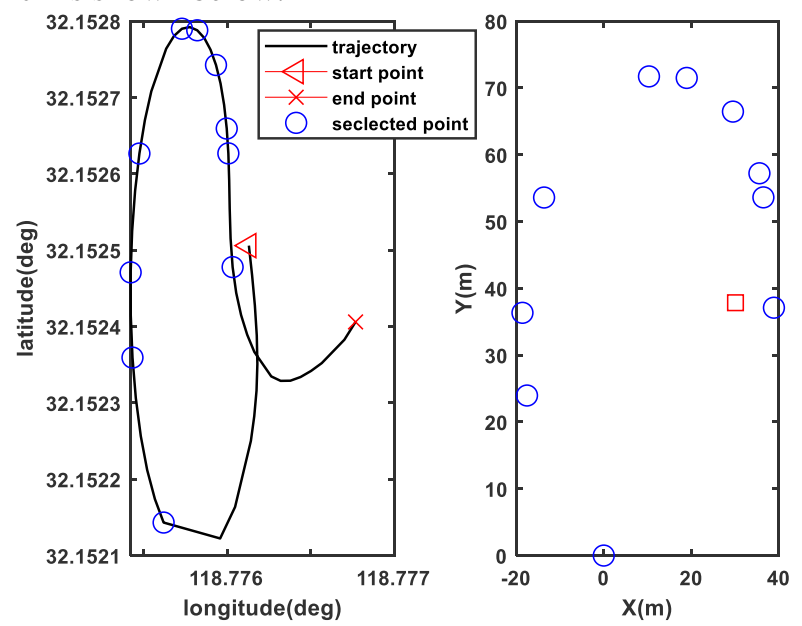

Fig. 15 the diagram of the localization scenario

Note that the estimation result of the source position will be different if different numbers of the ship position are selected. However, the results are convincing if all the algorithms are performed under the same sensor numbers. Only nine ship positions are selected to calculate the source position.

The source position error and bias norm with different algorithms are shown below.

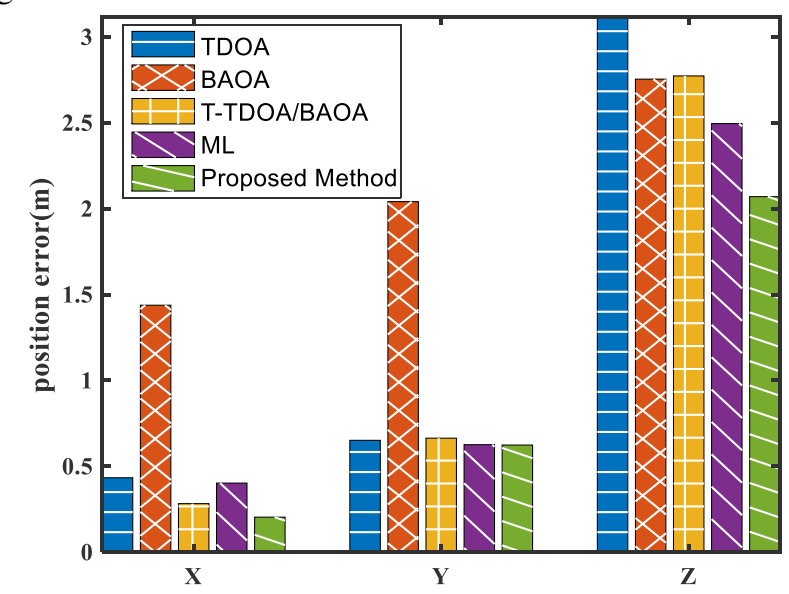

Fig. 16 Comparison of position error with different methods.

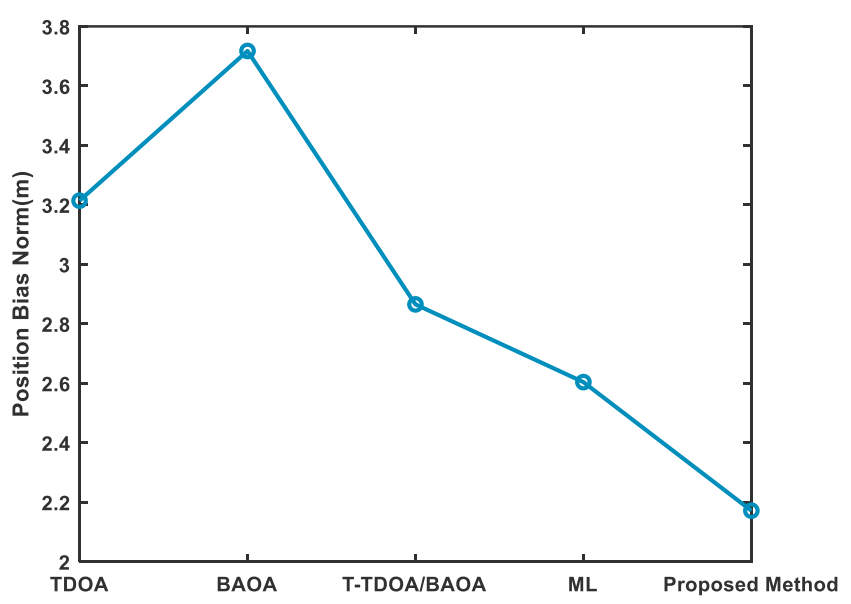

Fig. 17 Comparison of bias norm with different methods.

Fig. 16 shows the proposed method has the best estimation performance among these methods. In terms of the bias norm, the bias norm of the proposed method is $2.17 \mathrm{~m}$, which is smaller than the TDOA $(3.21 \mathrm{~m})$, BAOA $(3.72 \mathrm{~m})$, TTDOA/BAOA $(2.87 \mathrm{~m})$, and ML $(2.60 \mathrm{~m})$. This completes the proof that the proposed method has a higher position accuracy and smaller estimation bias than the traditional methods.

\section{II. $\quad$ Test 2}

A different ship trajectory is considered in this test. The ship moves slowly around the source. The trajectory is marked as the black line in Fig. 18. Several ship positions are selected as the reference to calculate the source, which is marked as a blue circle. In this test, the source is outside of the circle formed by the sensors. Take the first ship position as the reference position and show their positions in rectangular coordinates as the right picture in Fig. 18 shows. The true source position is marked as a red square, which is shown below.

Note that the estimation result of the source position will be different if different numbers of the ship position are selected. However, the results are convincing if all the algorithms are performed under the same sensor numbers. Only ten ship positions are selected to calculate the source position.
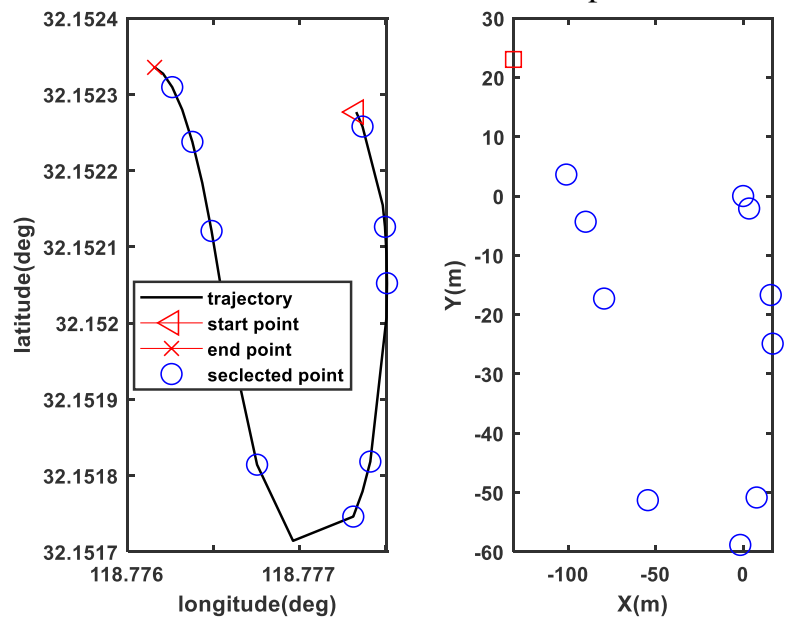

Fig. 18 the diagram of the localization scenario

The source position error and bias norm with different algorithms are shown below. 


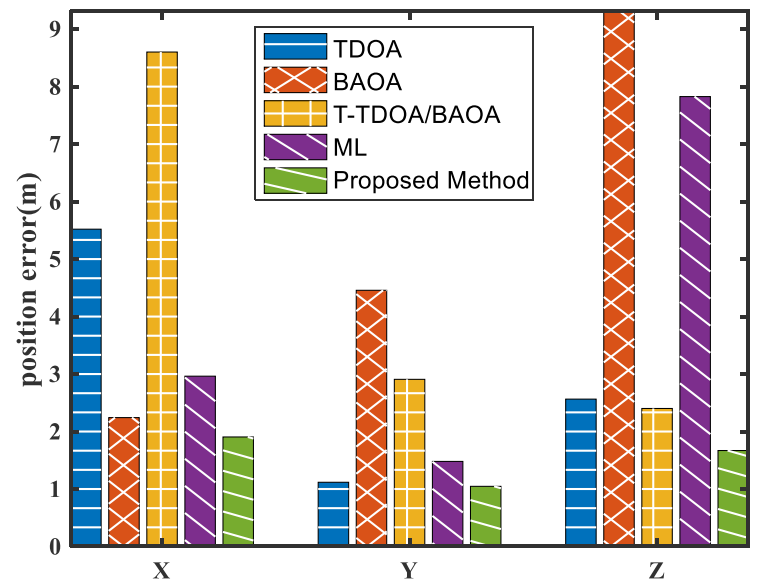

Fig. 19 Comparison of position error with different methods.

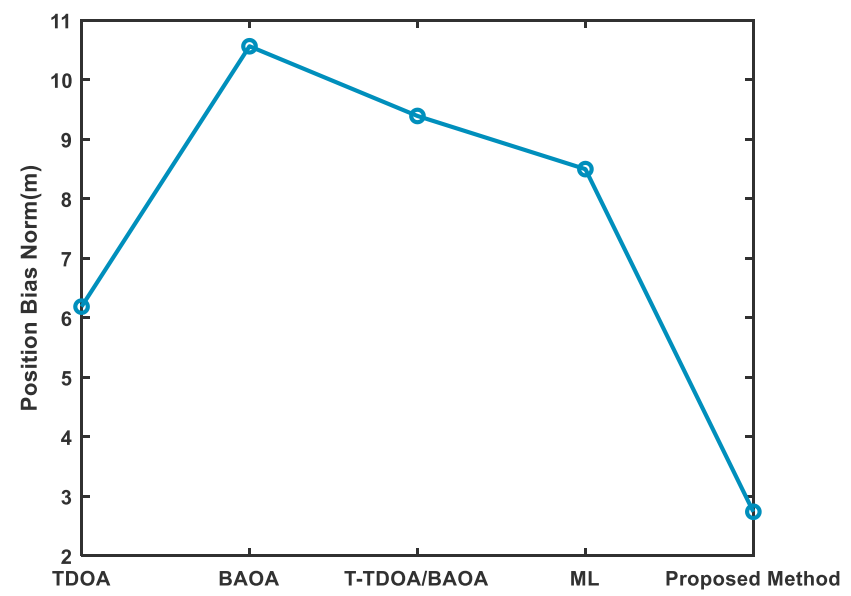

Fig. 20 Comparison of bias norm with different methods.

Fig. 19 shows the proposed method has the best estimation performance among these methods. In terms of the bias norm, the bias norm of the proposed method is $2.74 \mathrm{~m}$, which is smaller than the TDOA $(6.19 \mathrm{~m})$, BAOA $(10.56 \mathrm{~m}), \mathrm{T}$ TDOA/BAOA $(9.39 \mathrm{~m})$, and ML $(8.50 \mathrm{~m})$. This completes the proof that the proposed method has a higher position accuracy and smaller estimation bias than the traditional methods.

\section{FUTURE WORKS}

This paper focuses on the underwater acoustical localization problem based on the time difference and bearing measurements, which is first proposed in [2] using the onboard USBL system. Our future work includes studying underwater single-source passive navigation model with the onboard USBL system and applying the proposed algorithm to the passive inertial navigation system (INS) /TDOA/BAOA navigation model successfully.

The single-source navigation model is a passive navigation, where the underwater vehicles receive the time difference or the bearings sent by the sound source installed on the bottom of the sea to complete the positioning. It was named as single-source navigation as the positioning of the underwater vehicles can be accomplished with only one sound source. The detailed information about the single-source navigation model can be seen in [2]. The proposed method can be an appropriate solution for the single-source positioning.

The inertial navigation system can provide attitude, velocity and position information for the underwater vehicles. However, the errors of INS will accumulate and diverge with time. The solution of single-source positioning can correct the position error of INS.

Based on the completion of TDOA/BAOA localization, some key technologies need to be studied in the future. Considering the complex underwater environment, the robust TDOA/BAOA localization method should be further researched in the presence of outliers. Then the INS/TDOA/BAOA integrated navigation algorithm should be studied. It can provide high precision attitude and position information for the underwater vehicles.

\section{CONCLUSION}

We consider a localization scenario that the time difference and bearing information are used. The localization model is analyzed and the closed-form solution is developed. To improve the estimation accuracy in the case of large noise, an iterative constrained bias-reduction method is also designed. We have derived the CRLB of the hybrid TDOA/BAOA system. Several tests have been illustrated to verify the effectiveness of the proposed algorithm. The proposed method is proved to have better estimation accuracy than that of using TDOA only or BAOA only. It still outperforms the existing methods no matter the TDOA or BAOA noise changes. The proposed method can achieve the CRLB accuracy better. In the underwater experiment with the onboard USBL system, the algorithm is proved to have the best estimation performance and it is an efficient underwater acoustical localization method.

\section{APPENDIX A}

We will give the detailed derivation of $\frac{\partial \boldsymbol{m}^{o}}{\partial \boldsymbol{u}_{0}}$.

$$
\begin{aligned}
& \frac{\partial \boldsymbol{m}^{o}}{\partial \boldsymbol{u}_{0}}=\left[\begin{array}{l}
\frac{\partial \alpha^{o}}{\partial u_{0}} \\
\frac{\partial \beta^{o}}{\partial u_{0}}
\end{array}\right]
\end{aligned}
$$

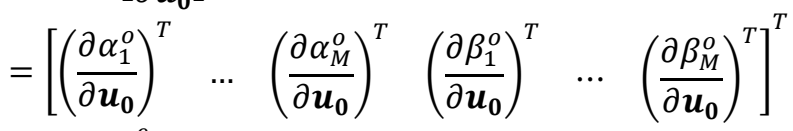

Take $\frac{\partial \alpha_{i}^{o}}{\partial \boldsymbol{u}_{\mathbf{0}}}$ as an example. $(i=1,2, \ldots M)$

$$
\frac{\partial \alpha_{i}^{o}}{\partial \boldsymbol{u}_{\mathbf{0}}}=\left[\begin{array}{lll}
\frac{\partial \alpha_{i}^{o}}{\partial \mathrm{x}} & \frac{\partial \alpha_{i}^{o}}{\partial \mathrm{y}} & \frac{\partial \alpha_{i}^{o}}{\partial \mathrm{z}}
\end{array}\right]
$$

where

$$
\begin{aligned}
& \frac{\partial \alpha_{i}^{o}}{\partial \mathrm{x}}=a\left(\frac{1}{\left\|u_{0}-s_{i}\right\|}-\frac{\left(x-x_{i}\right)^{2}}{\left(\left(u_{0}-s_{i}\right)^{T}\left(u_{0}-s_{i}\right)\right)^{1.5}}\right) . \\
& \frac{\partial \alpha_{i}^{o}}{\partial \mathrm{y}}=a\left(-\frac{\left(x-x_{i}\right)\left(\mathrm{y}-y_{i}\right)}{\left(\left(u_{0}-s_{i}\right)^{T}\left(u_{0}-s_{i}\right)\right)^{1.5}}\right) \\
& \frac{\partial \alpha_{i}^{o}}{\partial \mathrm{z}}=a\left(-\frac{\left(x-x_{i}\right)\left(\mathrm{z}-z_{i}\right)}{\left(\left(u_{0}-s_{i}\right)^{T}\left(u_{0}-s_{i}\right)\right)^{1.5}}\right) . \\
& a=\frac{-1}{\sqrt{1-\left(\frac{x-x_{i}}{\left\|u_{0}-s_{i}\right\|}\right)^{2}}} .
\end{aligned}
$$


Thus, $\frac{\partial \beta_{i}^{o}}{\partial \boldsymbol{u}_{0}}$ has the same form as $\frac{\partial \alpha_{i}^{o}}{\partial \boldsymbol{u}_{0}}$. It can be represented as.

$$
\frac{\partial \beta_{i}^{o}}{\partial \boldsymbol{u}_{\mathbf{0}}}=\left[\begin{array}{lll}
\frac{\partial \beta_{i}^{o}}{\partial \mathrm{x}} & \frac{\partial \beta_{i}^{o}}{\partial \mathrm{y}} & \frac{\partial \beta_{i}^{o}}{\partial \mathrm{z}}
\end{array}\right]
$$

where

$$
\begin{aligned}
& \frac{\partial \beta_{i}^{o}}{\partial \mathrm{x}}=b\left(-\frac{\left(x-x_{i}\right)\left(\mathrm{y}-y_{i}\right)}{\left(\left(u_{0}-s_{i}\right)^{T}\left(u_{0}-s_{i}\right)\right)^{1.5}}\right) . \\
& \frac{\partial \alpha_{i}^{o}}{\partial \mathrm{y}}=b\left(\frac{1}{\left\|u_{0}-s_{i}\right\|}-\frac{\left(y-y_{i}\right)^{2}}{\left(\left(u_{0}-s_{i}\right)^{T}\left(u_{0}-s_{i}\right)\right)^{1.5}}\right) . \\
& \frac{\partial \alpha_{i}^{o}}{\partial \mathrm{z}}=b\left(-\frac{\left(y-y_{i}\right)\left(\mathrm{z}-z_{i}\right)}{\left(\left(u_{0}-s_{i}\right)^{T}\left(u_{0}-s_{i}\right)\right)^{1.5}}\right) . \\
& b=\frac{-1}{\sqrt{1-\left(\frac{y-y_{i}}{\left\|u_{0}-s_{i}\right\|}\right)^{2}}} .
\end{aligned}
$$

\section{APPENDIX B}

Matrix B in (13) can be expressed as

$$
B=\left[\begin{array}{cc}
M_{1} & M_{12} \\
0 M-1 \times 2 M & M_{2}
\end{array}\right]
$$

where $\quad M_{1}=\left[\begin{array}{cc}\boldsymbol{B}_{21} & 0_{M \times M} \\ 0_{M \times M} & \boldsymbol{B}_{23}\end{array}\right], M_{12}=\left[\begin{array}{l}\boldsymbol{B}_{22} \\ \boldsymbol{B}_{24}\end{array}\right], M_{2}=\boldsymbol{B}_{1}$ $\boldsymbol{B}_{1}, \boldsymbol{B}_{21}, \boldsymbol{B}_{22}, \boldsymbol{B}_{23}, \boldsymbol{B}_{24}$ are defined in (13).

The inverse of matrix B can be expressed as.

$$
B^{-1}=\left[\begin{array}{cc}
M_{1}^{-1} & -M_{1}^{-1} M_{12} M_{2}^{-1} \\
0_{M-1 \times 2 M} & M_{2}^{-1}
\end{array}\right]
$$

Thus, $G_{4}$ can be expressed as follows

$$
\begin{aligned}
& G_{4}=\left[\begin{array}{cc}
M_{1}^{-1} & -M_{1}^{-1} M_{12} M_{2}^{-1} \\
0_{M-1 \times 2 M} & M_{2}^{-1}
\end{array}\right]\left[\begin{array}{l}
\boldsymbol{G}_{2} \\
\boldsymbol{G}_{\mathbf{1}}
\end{array}\right] \boldsymbol{B}_{3}^{-1} \boldsymbol{G}_{\mathbf{3}} \\
& =\left[\begin{array}{c}
M_{1}^{-1} \boldsymbol{G}_{2}-M_{1}^{-1} M_{12} M_{2}^{-1} \boldsymbol{G}_{\mathbf{1}} \\
M_{2}^{-1} \boldsymbol{G}_{\mathbf{1}}
\end{array}\right] \boldsymbol{B}_{3}^{-1} \boldsymbol{G}_{\mathbf{3}} \\
& =\left[\begin{array}{c}
M_{1}^{-1} G_{2} B_{3}^{-1} G_{3}-M_{1}^{-1} M_{12} M_{2}^{-1} G_{1} B_{3}^{-1} G_{3} \\
M_{2}^{-1} G_{1} B_{3}^{-1} G_{3}
\end{array}\right] \\
& =\left[\begin{array}{l}
G_{41} \\
G_{42}
\end{array}\right]
\end{aligned}
$$

where $G_{41}=M_{1}^{-1} \boldsymbol{G}_{\mathbf{2}} \boldsymbol{B}_{3}^{-1} \boldsymbol{G}_{\mathbf{3}}-M_{1}^{-1} M_{12} M_{2}^{-1} \boldsymbol{G}_{\mathbf{1}} \boldsymbol{B}_{3}^{-1} \boldsymbol{G}_{\mathbf{3}} \quad$ and $G_{42}=M_{2}^{-1} \boldsymbol{G}_{\mathbf{1}} \boldsymbol{B}_{3}^{-1} \boldsymbol{G}_{\mathbf{3}}$.

Put the definitions of $\boldsymbol{G}_{\mathbf{1}}, \boldsymbol{G}_{\mathbf{2}}, \boldsymbol{G}_{\mathbf{3}}$ and $\boldsymbol{B}_{\mathbf{3}}$ into $G_{41}$. The first and $\mathrm{M}+1$ rows of $G_{41}$ are

$$
\begin{gathered}
G_{41}(1,1)=\frac{1}{\left\|\boldsymbol{u}_{\mathbf{0}}-\boldsymbol{s}_{1}\right\| \sin \left(\alpha_{1}^{o}\right)}-\frac{\boldsymbol{\rho}_{\widetilde{\boldsymbol{u}}_{0}, s_{1}}^{T}(x) \cos \left(\alpha_{1}^{o}\right)}{\left\|\boldsymbol{u}_{0}-\boldsymbol{s}_{1}\right\| \sin \left(\alpha_{1}^{o}\right)} \\
G_{41}(1,2)=-\frac{\boldsymbol{\rho}_{\widetilde{\boldsymbol{u}}_{0}, s_{1}}^{T}(y) \cos \left(\alpha_{1}^{o}\right)}{\left\|\boldsymbol{u}_{\mathbf{0}}-\boldsymbol{s}_{1}\right\| \sin \left(\alpha_{1}^{o}\right)} \\
G_{41}(1,3)=-\frac{\boldsymbol{\rho}_{\widetilde{\boldsymbol{u}}_{0}, s_{1}}^{T}(z) \cos \left(\alpha_{1}^{o}\right)}{\left\|\boldsymbol{u}_{\mathbf{0}}-\boldsymbol{s}_{1}\right\| \sin \left(\alpha_{1}^{o}\right)} \\
G_{41}(M+1,1)=-\frac{\boldsymbol{\rho}_{\widetilde{\boldsymbol{u}}_{0}, s_{1}}^{T}(x) \cos \left(\beta_{1}^{o}\right)}{\left\|\boldsymbol{u}_{0}-\boldsymbol{s}_{1}\right\| \sin \left(\beta_{1}^{o}\right)} \\
G_{41}(M+1,2)=\frac{1}{\left\|\boldsymbol{u}_{\mathbf{0}}-\boldsymbol{s}_{1}\right\| \sin \left(\beta_{1}^{o}\right)}-\frac{\boldsymbol{\rho}_{\widetilde{\boldsymbol{u}}_{0}, s_{1}}^{T}(y) \cos \left(\beta_{1}^{o}\right)}{\left\|\boldsymbol{u}_{\mathbf{0}}-\boldsymbol{s}_{1}\right\| \sin \left(\beta_{1}^{o}\right)} \\
G_{41}(M+1,3)=-\frac{\boldsymbol{\rho}_{\widetilde{\boldsymbol{u}}_{0}, s_{1}}^{T}(z) \cos \left(\beta_{1}^{o}\right)}{\left\|\boldsymbol{u}_{0}-\boldsymbol{s}_{1}\right\| \sin \left(\beta_{1}^{o}\right)}
\end{gathered}
$$

where $\boldsymbol{\rho}_{\widetilde{u}_{0}, s_{1}}^{T}(x)=\frac{x-x_{1}}{\left\|\widetilde{u}_{0}-s_{1}\right\|}, \boldsymbol{\rho}_{\widetilde{u}_{0}, s_{1}}^{T}(y)=\frac{y-y_{1}}{\left\|\widetilde{u}_{0}-s_{1}\right\|}, \boldsymbol{\rho}_{\widetilde{u}_{0}, s_{1}}^{T}(z)=$ $\frac{z-z_{1}}{\left\|\widetilde{u}_{0}-s_{1}\right\|}$.

The $i$ th rows $(i=2, \ldots M)$ of $G_{41}$ is

$$
\begin{aligned}
& G_{41}(i, 1)=\frac{1}{\left\|\boldsymbol{u}_{0}-\boldsymbol{s}_{i}\right\| \sin \left(\alpha_{i}^{o}\right)}-\frac{\cos \left(\alpha_{i}^{o}\right)\left(x_{1}-x_{i}\right)}{\left\|\boldsymbol{u}_{0}-\boldsymbol{s}_{i}\right\|^{2} \sin \left(\alpha_{i}^{o}\right)} \\
& -\boldsymbol{\rho}_{\widetilde{\boldsymbol{u}}_{0}, \boldsymbol{s}_{1}}^{T}(x)\left[\frac{\cos \left(\alpha_{i}^{o}\right)}{\left\|\boldsymbol{u}_{\mathbf{0}}-\boldsymbol{s}_{i}\right\| \sin \left(\alpha_{i}^{o}\right)}-\frac{r_{i 1}^{0} \cos \left(\alpha_{i}^{o}\right)}{\left\|\boldsymbol{u}_{\mathbf{0}}-\boldsymbol{s}_{i}\right\|^{2} \sin \left(\alpha_{i}^{o}\right)}\right] \\
& G_{41}(i, 2)=-\frac{\cos \left(\alpha_{i}^{o}\right)\left(y_{1}-y_{i}\right)}{\left\|\boldsymbol{u}_{\mathbf{0}}-\boldsymbol{s}_{i}\right\|^{2} \sin \left(\alpha_{i}^{o}\right)} \\
& -\boldsymbol{\rho}_{\widetilde{\boldsymbol{u}}_{0}, \boldsymbol{s}_{1}}^{T}(y)\left[\frac{\cos \left(\alpha_{i}^{o}\right)}{\left\|\boldsymbol{u}_{\mathbf{0}}-\boldsymbol{s}_{i}\right\| \sin \left(\alpha_{i}^{o}\right)}-\frac{r_{i 1}^{0} \cos \left(\alpha_{i}^{o}\right)}{\left\|\boldsymbol{u}_{\mathbf{0}}-\boldsymbol{s}_{i}\right\|^{2} \sin \left(\alpha_{i}^{o}\right)}\right] \\
& G_{41}(i, 3)=-\frac{\cos \left(\alpha_{i}^{o}\right)\left(z_{1}-z_{i}\right)}{\left\|\boldsymbol{u}_{\mathbf{0}}-\boldsymbol{s}_{i}\right\|^{2} \sin \left(\alpha_{1}^{o}\right)} \\
& -\boldsymbol{\rho}_{\tilde{\boldsymbol{u}}_{0}, \boldsymbol{s}_{1}}^{T}(z)\left[\frac{\cos \left(\alpha_{i}^{o}\right)}{\left\|\boldsymbol{u}_{\mathbf{0}}-\boldsymbol{s}_{i}\right\| \sin \left(\alpha_{i}^{o}\right)}-\frac{r_{i 1}^{0} \cos \left(\alpha_{i}^{o}\right)}{\left\|\boldsymbol{u}_{\mathbf{0}}-\boldsymbol{s}_{i}\right\|^{2} \sin \left(\alpha_{i}^{o}\right)}\right] \\
& \text { The } j \text { th rows }(j=M+1, \ldots 2 M) \text { of } G_{41} \text { is } \\
& G_{41}(j, 1)=-\frac{\cos \left(\beta_{i}^{o}\right)\left(x_{1}-x_{i}\right)}{\left\|\boldsymbol{u}_{\mathbf{0}}-\boldsymbol{s}_{i}\right\|^{2} \sin \left(\beta_{i}^{o}\right)} \\
& -\boldsymbol{\rho}_{\tilde{\boldsymbol{u}}_{0}, \boldsymbol{s}_{1}}^{T}(x)\left[\frac{\cos \left(\beta_{i}^{o}\right)}{\left\|\boldsymbol{u}_{\mathbf{0}}-\boldsymbol{s}_{i}\right\| \sin \left(\beta_{i}^{o}\right)}-\frac{r_{i 1}^{0} \cos \left(\beta_{i}^{o}\right)}{\left\|\boldsymbol{u}_{\mathbf{0}}-\boldsymbol{s}_{i}\right\|^{2} \sin \left(\beta_{i}^{o}\right)}\right] \\
& G_{41}(j, 2)=\frac{1}{\left\|\boldsymbol{u}_{\mathbf{0}}-\boldsymbol{s}_{i}\right\| \sin \left(\beta_{i}^{o}\right)}-\frac{\cos \left(\beta_{i}^{o}\right)\left(y_{1}-y_{i}\right)}{\left\|\boldsymbol{u}_{\mathbf{0}}-\boldsymbol{s}_{i}\right\|^{2} \sin \left(\beta_{i}^{o}\right)} \\
& -\boldsymbol{\rho}_{\widetilde{\boldsymbol{u}}_{0}, \boldsymbol{s}_{1}}^{T}(y)\left[\frac{\cos \left(\beta_{i}^{o}\right)}{\left\|\boldsymbol{u}_{\mathbf{0}}-\boldsymbol{s}_{i}\right\| \sin \left(\beta_{i}^{o}\right)}-\frac{r_{i 1}^{0} \cos \left(\beta_{i}^{o}\right)}{\left\|\boldsymbol{u}_{\mathbf{0}}-\boldsymbol{s}_{i}\right\|^{2} \sin \left(\beta_{i}^{o}\right)}\right] \\
& G_{41}(j, 3)=-\frac{\cos \left(\beta_{i}^{o}\right)\left(z_{1}-z_{i}\right)}{\left\|\boldsymbol{u}_{\mathbf{0}}-\boldsymbol{s}_{i}\right\|^{2} \sin \left(\beta_{i}^{o}\right)} \\
& -\boldsymbol{\rho}_{\widetilde{\boldsymbol{u}}_{0}, \boldsymbol{s}_{1}}^{T}(z)\left[\frac{\cos \left(\beta_{i}^{o}\right)}{\left\|\boldsymbol{u}_{\mathbf{0}}-\boldsymbol{s}_{i}\right\| \sin \left(\beta_{i}^{o}\right)}-\frac{r_{i 1}^{0} \cos \left(\beta_{i}^{o}\right)}{\left\|\boldsymbol{u}_{\mathbf{0}}-\boldsymbol{s}_{i}\right\|^{2} \sin \left(\beta_{i}^{o}\right)}\right]
\end{aligned}
$$

\section{APPENDIX C}

Put (44) and the definitions of $\boldsymbol{\rho}_{\tilde{\boldsymbol{u}}_{0}, s_{1}}^{T}$ into $G_{41}$. The $i$ th rows of $G_{41}$ can be expressed as follows. $(i=2, \ldots M)$.

$$
\begin{aligned}
& G_{41}(i, 1)=\frac{1}{\left\|\boldsymbol{u}_{\mathbf{0}}-\boldsymbol{s}_{i}\right\| \sin \left(\alpha_{i}^{o}\right)}-\frac{\cos \left(\alpha_{i}^{o}\right)\left(x_{1}-x_{i}\right)}{\left\|\boldsymbol{u}_{\mathbf{0}}-\boldsymbol{s}_{i}\right\|^{2} \sin \left(\alpha_{i}^{o}\right)} \\
& -\boldsymbol{\rho}_{\widetilde{\boldsymbol{u}}_{0}, \boldsymbol{s}_{1}}^{T}(x)\left[\frac{\cos \left(\alpha_{i}^{o}\right)}{\left\|\boldsymbol{u}_{0}-\boldsymbol{s}_{i}\right\| \sin \left(\alpha_{i}^{o}\right)}-\frac{r_{i 1}^{0} \cos \left(\alpha_{i}^{o}\right)}{\left\|\boldsymbol{u}_{0}-\boldsymbol{s}_{i}\right\|^{2} \sin \left(\alpha_{i}^{o}\right)}\right] \\
& =\frac{1}{r_{i} \sqrt{1-\left(\frac{x-x_{i}}{r_{i}}\right)^{2}}}-\frac{\frac{\left(x-x_{i}\right)}{r_{i}}\left(x_{1}-x_{i}\right)}{r_{i}^{2} \sqrt{1-\left(\frac{x-x_{i}}{r_{i}}\right)^{2}}} \\
& -\frac{x-x_{1}}{r_{1}}\left[\frac{\frac{\left(x-x_{i}\right)}{r_{i}}}{r_{i} \sqrt{1-\left(\frac{x-x_{i}}{r_{i}}\right)^{2}}}-\frac{\left(r_{i}-r_{1}\right) \frac{\left(x-x_{i}\right)}{r_{i}}}{r_{i}^{2} \sqrt{1-\left(\frac{x-x_{i}}{r_{i}}\right)^{2}}}\right] \\
& =\frac{1}{\sqrt{1-\left(\frac{x-x_{i}}{r_{i}}\right)^{2}}}\left[\frac{1}{r_{i}}-\frac{\left(x_{1}-x_{i}\right)\left(x-x_{i}\right)}{r_{i}^{3}}\right. \\
& -\frac{\left(x-x_{1}\right)}{r_{1}} \frac{\left(x-x_{i}\right)}{r_{i}^{2}} \\
& +r_{i} \frac{x-x_{1}}{r_{1}} \frac{\left(x-x_{i}\right)}{r_{i}^{3}} \\
& \left.-r_{1} \frac{x-x_{1}}{r_{1}} \frac{\left(x-x_{i}\right)}{r_{i}^{3}}\right]
\end{aligned}
$$




$$
\begin{aligned}
& =\frac{1}{\sqrt{1-\left(\frac{x-x_{i}}{r_{i}}\right)^{2}}}\left[\frac{1}{r_{i}}-\frac{\left(x-x_{i}\right)^{2}}{r_{i}^{3}}\right] \\
& \text { Thus, } G_{41}(i, 2) \text { and } G_{41}(i, 3) \text { can be obtained. } \\
& G_{41}(i, 2)=-\frac{\cos \left(\alpha_{i}^{o}\right)\left(y_{1}-y_{i}\right)}{\left\|\boldsymbol{u}_{0}-\boldsymbol{s}_{i}\right\|^{2} \sin \left(\alpha_{i}^{o}\right)} \\
& -\boldsymbol{\rho}_{\widetilde{\boldsymbol{u}}_{\mathbf{0}}, \boldsymbol{s}_{1}}^{T}(y)\left[\frac{\cos \left(\alpha_{i}^{o}\right)}{\left\|\boldsymbol{u}_{\mathbf{0}}-\boldsymbol{s}_{i}\right\| \sin \left(\alpha_{i}^{o}\right)}-\frac{r_{i 1}^{0} \cos \left(\alpha_{i}^{o}\right)}{\left\|\boldsymbol{u}_{\mathbf{0}}-\boldsymbol{s}_{i}\right\|^{2} \sin \left(\alpha_{i}^{o}\right)}\right] \\
& =\frac{1}{\sqrt{1-\left(\frac{x-x_{i}}{r_{i}}\right)^{2}}}\left[-\frac{\left(x-x_{i}\right)\left(y-y_{i}\right)}{r_{i}^{3}}\right] \\
& G_{41}(i, 3)=-\frac{\cos \left(\alpha_{i}^{o}\right)\left(z_{1}-z_{i}\right)}{\left\|\boldsymbol{u}_{0}-\boldsymbol{s}_{i}\right\|^{2} \sin \left(\alpha_{i}^{o}\right)} \\
& -\boldsymbol{\rho}_{\widetilde{\boldsymbol{u}}_{\mathbf{0}}, \boldsymbol{s}_{1}}^{T}(z)\left[\frac{\cos \left(\alpha_{i}^{o}\right)}{\left\|\boldsymbol{u}_{\mathbf{0}}-\boldsymbol{s}_{i}\right\| \sin \left(\alpha_{i}^{o}\right)}-\frac{r_{i 1}^{0} \cos \left(\alpha_{i}^{o}\right)}{\left\|\boldsymbol{u}_{\mathbf{0}}-\boldsymbol{s}_{i}\right\|^{2} \sin \left(\alpha_{i}^{o}\right)}\right] \\
& =\frac{1}{\sqrt{1-\left(\frac{x-x_{i}}{r_{i}}\right)^{2}}}\left[-\frac{\left(x-x_{i}\right)\left(z-z_{i}\right)}{r_{i}^{3}}\right]
\end{aligned}
$$

\section{REFERENCES}

[1] A. Beck, P. Stoica, and J. Li, "Exact and approximate solutions of source localization problems," IEEE Trans. Signal Process., vol. 56, no. 5, pp. 1770-1778, May 2008.

[2] B. Jin, X. Xu, Y. Zhu, T. Zhang, and Q. Fei, "Single-Source Aided SemiAutonomous Passive Location for Correcting the Position of an Underwater Vehicle," in IEEE Sensors Journal, vol. 19, no. 9, pp. 32673275, 1 May 2019.

[3] R. Amiri, F. Behnia, and A. Noroozi, "An Efficient Estimator for TDOABased Source Localization With Minimum Number of Sensors," in IEEE Communications Letters, vol. 22, no. 12, pp. 2499-2502, Dec. 2018, DOI: 10.1109/LCOMM.2018.2876525.

[4] G. Wang and K. C. Ho, "Convex Relaxation Methods for Unified NearField and Far-Field TDOA-Based Localization," in IEEE Transactions on Wireless Communications, vol. 18, no. 4, pp. 2346-2360, April 2019, DOI: 10.1109/TWC.2019.2903037.

[5] M. R. Gholami, S. Gezici, and E. G. Strom, "TDOA Based Positioning in the Presence of Unknown Clock Skew," in IEEE Transactions on Communications, vol. 61, no. 6, pp. 2522-2534, June 2013.

[6] Y. Sun, F. Zhang and Q. Wan, "Wireless Sensor Network-Based Localization Method Using TDOA Measurements in MPR," in IEEE Sensors Journal, vol. 19, no. 10, pp. 3741-3750, 15 May 15, 2019, DOI: 10.1109/JSEN.2019.2892652.

[7] G. Wang, H. Chen, Y. Li and N. Ansari, "NLOS Error Mitigation for TOA-Based Localization via Convex Relaxation," in IEEE Transactions on Wireless Communications, vol. 13, no. 8, pp. 4119-4131, Aug. 2014, DOI: 10.1109/TWC.2014.2314640.

[8] S. Gao, F. Zhang, and G. Wang, "NLOS Error Mitigation for TOA-Based Source Localization With Unknown Transmission Time," in IEEE Sensors Journal, vol. 17, no. 12, pp. 3605-3606, 15 June 15, 2017, DOI: 10.1109/JSEN.2017.2698073.

[9] Y. Zheng, M. Sheng, J. Liu, and J. Li, "Exploiting AoA Estimation Accuracy for Indoor Localization: A Weighted AoA-Based Approach," in IEEE Wireless Communications Letters, vol. 8, no. 1, pp. 65-68, Feb. 2019, DOI: 10.1109/LWC.2018.2853745.

[10] H. Shao, X. Zhang and Z. Wang, "Efficient Closed-Form Algorithms for AOA Based Self-Localization of Sensor Nodes Using Auxiliary Variables," in IEEE Transactions on Signal Processing, vol. 62, no. 10, pp. 2580-2594, May 15, 2014, DOI: 10.1109/TSP.2014.2314064.

[11] S. Sun, X. Zhang, C. Zheng, J. Fu, and C. Zhao, "Underwater Acoustical Localization of the Black Box Utilizing Single Autonomous Underwater Vehicle Based on the Second-Order Time Difference of Arrival," in IEEE Journal of Oceanic Engineering, DOI: 10.1109/JOE.2019.2950954.

[12] Y. T. Chan and K. C. Ho, "A simple and efficient estimator for hyperbolic location," in IEEE Transactions on Signal Processing, vol. 42, no. 8, pp. 1905-1915, Aug. 1994.
[13] A. Noroozi, A. H. Oveis, S. M. Hosseini, and M. A. Sebt, "Improved Algebraic Solution for Source Localization From TDOA and FDOA Measurements," in IEEE Wireless Communications Letters, vol. 7, no. 3, pp. 352-355, June 2018, DOI: 10.1109/LWC.2017.2777995.

[14] S. Cao, X. Chen, X. Zhang and X. Chen, "Combined Weighted Method for TDOA-Based Localization," in IEEE Transactions on Instrumentation and Measurement, vol. 69, no. 5, pp. 1962-1971, May 2020, DOI: 10.1109/TIM.2019.2921439.

[15] Y. Wang and K. C. Ho, "An Asymptotically Efficient Estimator in Closed-Form for 3-D AOA Localization Using a Sensor Network," in IEEE Transactions on Wireless Communications, vol. 14, no. 12, pp. 6524-6535, Dec. 2015, DOI: 10.1109/TWC.2015.2456057.

[16] Y. Sun, K. C. Ho, and Q. Wan, "Eigenspace Solution for AOA Localization in Modified Polar Representation," in IEEE Transactions on Signal Processing, vol. 68, pp. 2256-2271, 2020, DOI: 10.1109/TSP.2020.2981773.

[17] Li Cong and Weihua Zhuang, "Hybrid TDOA/AOA mobile user location for wideband CDMA cellular systems," in IEEE Transactions on Wireless Communications, vol. 1, no. 3, pp. 439-447, July 2002, DOI: 10.1109/TWC.2002.800542.

[18] Bishop A N, Fidan B, Doanay K, et al. Exploiting geometry for improved hybrid AOA/TDOA-based localization. in Signal Processing, vol.88, no.7, pp:1775-1791, 2008.

[19] J. Yin, Q. Wan, S. Yang, and K. C. Ho, "A Simple and Accurate TDOAAOA Localization Method Using Two Stations," in IEEE Signal Processing Letters, vol. 23, no. 1, pp. 144-148, Jan. 2016, DOI: 10.1109/LSP.2015.2505138.

[20] Y. Wang and K. C. Ho, "Unified Near-Field and Far-Field Localization for AOA and Hybrid AOA-TDOA Positionings," in IEEE Transactions on Wireless Communications, vol. 17, no. 2, pp. 1242-1254, Feb. 2018, DOI: 10.1109/TWC.2017.2777457.

[21] L. Zhang, T. Zhang, J. Tong, C. Weng, and Y. Li. "A calibration method of ultra-short baseline installation error with large misalignment based on variational Bayesian unscented Kalman filter" Rev. Sci. Instrum. vol.90, 055003,2019 .

[22] J. Wang, T. Zhang, B. Jin, Y. Zhu, and J. Tong, "Student's t-Based Robust Kalman Filter for a SINS/USBL Integration Navigation Strategy," in IEEE Sensors Journal, vol. 20, no. 10, pp. 5540-5553, 15 May 15, 2020.

[23] H. Yu, G. Huang, J. Gao, and B. Liu, "An Efficient Constrained Weighted Least Squares Algorithm for Moving Source Location Using TDOA and FDOA Measurements," in IEEE Transactions on Wireless Communications, vol. 11, no. 1, pp. 44-47, January 2012.

[24] X. Qu, L. Xie, and W. Tan, "Iterative Constrained Weighted Least Squares Source Localization Using TDOA and FDOA Measurements," in IEEE Transactions on Signal Processing, vol. 65, no. 15, pp. 3990-4003, 1 Aug.1, 2017, DOI: 10.1109/TSP.2017.2703667.

[25] Y. Wang and K. C. Ho, "TDOA Positioning Irrespective of Source Range," in IEEE Transactions on Signal Processing, vol. 65, no. 6, pp. 1447-1460, 15 March 15, 2017, DOI: 10.1109/TSP.2016.2630030.

[26] K. C. Ho, "Bias Reduction for an Explicit Solution of Source Localization Using TDOA," in IEEE Transactions on Signal Processing, vol. 60, no. 5, pp. 2101-2114, May 2012.

[27] C. Zheng, 'Application of USBL Positioning Technology on Underwater Submersible Interfacing' Ph.D. dissertation, Harbin Engineering University, Harbin, China,2007.

[28] Y. Liu, F. Guo, L. Yang and W. Jiang, "An Improved Algebraic Solution for TDOA Localization With Sensor Position Errors," in IEEE Communications Letters, vol. 19, no. 12, pp. 2218-2221, Dec. 2015, DOI: 10.1109/LCOMM.2015.2486769.

[29] T. Sun and C. Dong, "Efficient source positioning method based on two stations using GROA and AOA measurements," in IET Signal Processing, vol. 14, no. 2, pp. 56-63, 4 2020, DOI: 10.1049/iet-spr.2019.0003.

[30] Y. Ji, C. Yu and B. D. O. Anderson, "Systematic Bias Correction in Source Localization," in IEEE Transactions on Aerospace and Electronic Systems, vol. 49, no. 3, pp. 1692-1709, July 2013.

[31] T. Zhang, L. Zhang, and H. Shin, "A novel and robust calibration method for the underwater transponder position," in IEEE Transactions on Instrumentation and Measurement, DOI: 10.1109/TIM.2020.3011540. 


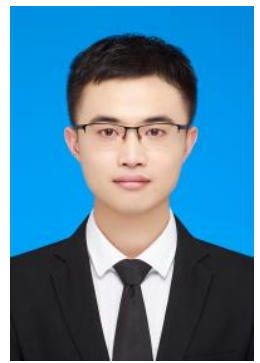

Liang Zhang received the M.S. degree in Navigation, Guidance, and Control from Nanjing University of Aeronautics and Astronautics, Nanjing, Jiangsu, in 2017. He is currently pursuing a Ph.D. degree in instrument science and technology at Southeast University, Nanjing, Jiangsu. His research interest includes inertial navigation, integrated navigation technology, and AUV underwater positioning technology.

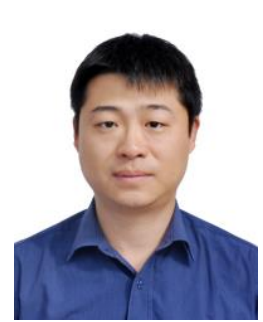

Tao Zhang received the Ph.D. degree in precision instrument and machinery from Southeast University, Nanjing, China, in 2008. He is currently a Professor with the School of Instrument Science and Engineering, Southeast University. His research interests include inertial navigation, AUV underwater positioning, and integrated navigation.

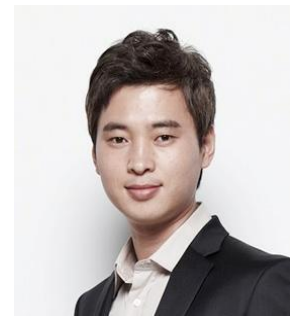

Hyo-Sang Shin received his BSc from Pusan National University in 2004 and gained an MSc on flight dynamics, guidance, and control in Aerospace Engineering from KAIST and a Ph.D. on cooperative missile guidance from Cranfield University in 2006 and 2010, respectively. He is currently a Professor on Guidance, Control and Navigation Systems in Autonomous and Intelligent Systems Group at Cranfield University. His current research interests include multiple target tracking, adaptive and sensor-based control, and distributed control of multiple agent systems.

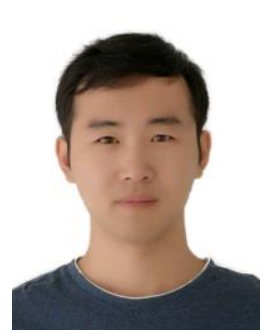

Xiang Xu was born in Nantong, Jiangsu, in 1988. He received the M.S. degree from Harbin Engineering University, Harbin, China, in 2014, and the Ph.D. degree from Southeast University, Nanjing, China, in 2018. He is currently a lecturer with the School of Electronics and Information Engineering, Soochow University, Suzhou, China. His research interests include initial alignment for inertial navigation, integrated navigation for an autonomous underwater vehicle, attitude estimation for lowcost inertial measurement unit, and information fusion. 
2020-12-16

\section{Efficient underwater acoustical localization method based on time difference and bearing measurements}

\section{Zhang, Liang}

IEEE

Zhang L, Zhang T, Shin H-S, Xu X. (2021) Efficient underwater acoustical localization method based on time difference and bearing measurements. IEEE Transactions on Instrumentation and Measurement, Volume 70, 2021, Article number 8501316

https://doi.org/10.1109/TIM.2020.3045193

Downloaded from Cranfield Library Services E-Repository 